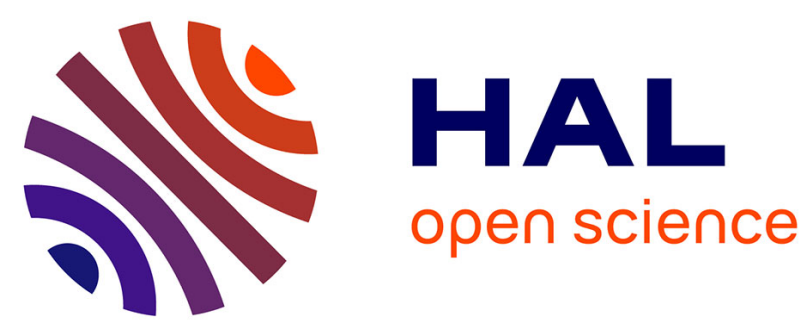

\title{
Asymptotic behavior and numerical simulations for an infection load-structured epidemiological model; Application to the transmission of prion pathologies
}

Antoine Perasso, Ulrich Razafison

\section{- To cite this version:}

Antoine Perasso, Ulrich Razafison. Asymptotic behavior and numerical simulations for an infection load-structured epidemiological model; Application to the transmission of prion pathologies. SIAM Journal on Applied Mathematics, 2014, 74 (5), pp.1571-1597. 10.1137/130946058 . hal-00907819v2

HAL Id: hal-00907819

https://hal.science/hal-00907819v2

Submitted on 11 Mar 2014

HAL is a multi-disciplinary open access archive for the deposit and dissemination of scientific research documents, whether they are published or not. The documents may come from teaching and research institutions in France or abroad, or from public or private research centers.
L'archive ouverte pluridisciplinaire HAL, est destinée au dépôt et à la diffusion de documents scientifiques de niveau recherche, publiés ou non, émanant des établissements d'enseignement et de recherche français ou étrangers, des laboratoires publics ou privés. 


\title{
ASYMPTOTIC BEHAVIOR AND NUMERICAL SIMULATIONS FOR AN INFECTION LOAD-STRUCTURED EPIDEMIOLOGICAL MODEL ; APPLICATION TO THE TRANSMISSION OF PRION PATHOLOGIES
}

\author{
ANTOINE PERASSO* AND ULRICH RAZAFISON ${ }^{\dagger}$
}

\begin{abstract}
In this article is studied an infection load-structured SI model with exponential growth of the infection, that incorporates a potential external source of contamination. We perform the analysis of the time asymptotic behavior of the solution by exhibiting epidemiological thresholds, such as the basic reproduction number, that ensure extinction or persistence of the disease in the contagion process. Moreover, a numerical scheme adapted to the model is developped and analyzed. This scheme is then used to illustrate the model with numerical simulations, applying this last to the transmission of prion pathologies.
\end{abstract}

Key words. Structured population dynamics, epidemic models, transport equations, nonlinear PDE, stability analysis, finite volume method

AMS subject classifications. 35B40, 65M08, 92D30, 92D40

1. Introduction. Epidemiological compartmental models of SIR type have been studied and extended in several directions since the classical Kermack and McKendrick [22, 23, 24] model was built. The goal of such epidemiological models is to describe the propagation of an infectious disease in a population, by incorporating in the model a susceptible class $S$ linked to an infective class $I$ and potentially a recovered class $R$. Various SIR epidemics models are described either by ordinary differential equations (ODEs) or by partial differential equations (PDEs), these latter models being structured according to other variables than the time (age, space...). We refer to Bailey [5], Brauer and Castillo-Chavez [6], Capasso [9], Diekmann and Heesterbeck [13], Murray [29], Perthame [34] and Thieme [43] and references therein for a presentation and examples of SIR models described by ODEs or PDEs.

This article is devoted to the analysis of asymptotic properties and to the analysis of a numerical scheme for an infection load-structured epidemiological SI model that describes an infection process with an exponential growth of a fatal-issued disease. As illustrated at the end of the article, such a structuration allows to model the transmission of prion pathologies (BSE, scrapie...).

Denoting by $i$ the infection load in the infective class, it is supposed that, identically to size-structured models (see Arino [4], Cushing [11], Webb [48] and references therein), the following evolution equation is satisfied with respect to time $t \geq 0$,

$$
\frac{d i}{d t}=\nu i
$$

where the positive constant $\nu$ denotes the growth velocity of the infection. The model also incorporates, additionnaly to the contagion process, a potential external source of contamination that may affect the population, that is supposed to be constant and permanent. Taking into account a mass action law as in SI models, the infection process is then described by a nonlinear system of ODE and PDE of transport type given for $t \geq 0$ and $i \in J=\left(i^{-},+\infty\right) \subset \mathbb{R}^{+}$by

$$
\left\{\begin{array}{l}
\frac{d S(t)}{d t}=\gamma-\left(\mu_{0}+\alpha\right) S(t)-S(t) \mathcal{T}(\beta I)(t), \quad t \geq 0, \\
\frac{\partial I(t, i)}{\partial t}=-\frac{\partial(\nu i I)(t, i)}{\partial i}-\mu(i) I(t, i)+\Phi(i) S(t) \mathcal{T}(\beta I)(t), \quad t \geq 0, i \in J \\
\nu i^{-} I\left(t, i^{-}\right)=\alpha S(t), \\
S(0)=S_{0} \in \mathbb{R}_{+}, \quad I(0, \cdot)=I_{0} \in L_{+}^{1}(J) .
\end{array}\right.
$$

In Problem (1.2), $\mathcal{T}$ is the integral operator defined for some integrable function $h$ on $J$ by

$$
\mathcal{T}: h \mapsto \int_{J} h(i) d i
$$

*UMR6249 Chrono-environnement - Université de Franche-Comté, antoine.perasso@univ-fcomte.fr

†UMR 6623 Laboratoire de Mathématiques de Besançon - Université de Franche-Comté, ulrich.razafison@univ-fcomte.fr 
As a consequence, $S(t)+\mathcal{T}(I)(t)$ denotes the total population at time $t \geq 0$, with initial population $S_{0}+\mathcal{T}\left(I_{0}\right)$

Throughout the article the following hypotheses are made on the model:

(i) $\mu_{0}, \nu>0$ and $\alpha, \gamma \geq 0$;

(ii) function $\Phi \in C^{\infty}(J)$ is a non negative function such that $\lim _{i \rightarrow+\infty} \Phi(i)=0$ and $\int_{J} \Phi(i) d i=1$;

(iii) functions $\mu, \beta \in L^{\infty}(J)$ are such that $\mu(i) \geq \mu_{0}$ and $\beta(i) \geq \beta_{0}>0$ for almost every (f.a.e) $i \in J$. As shown in the second equation, Problem (1.2) incorporates the infection load-structure $i \in J=$ $\left(i^{-},+\infty\right)$ of the infected population, with $i^{-}$as minimal infection load: the infimum $i^{-}$is a threshold in the evolution of the infection process from which the individuals are considered to be infected and infectious, so appear in the infective class $I$.

The model also takes into account demographics of the studied population by assigning a constant mortality rate $\mu_{0}$ and a constant entering flux $\gamma$ into the susceptible class $S$. The mortality rate for the infective class is an infection-load dependent function $i \mapsto \mu(i)$. A consequence of the assumption (iii) is that function $\mu$ satisfies the following asymptotic property,

$$
\lim _{i \rightarrow+\infty} \int_{i^{-}}^{i} \mu(s) d s=+\infty
$$

The limit in equation (1.3) models that all the infected individuals leave the stage $I$ by dying of the disease with a finite infection load.

The infectious process is due to an horizontal transmission of the disease, that is modeled with a mass action law of SI type, where the force of infection is given by $\mathcal{T}(\beta I)$ with $\beta$ transmission rate. When infection occurs, individuals get variable initial infection loads taking values in $J$. This variability of the initial infected load at the contamination is therefore represented by the probability density function $\Phi$, whose role is to attribute an initial infection load to susceptible individuals when they get infected. Such an approach has been developed for modelling the transmission of some prion pathologies[31, 41, 45]. In the model is also considered a potential external contamination source, supposed to be constant in time, that is modeled as an input into the system that affects susceptibles with a constant rate $\alpha$ by attributing the minimal initial infection load $i^{-}$when the contamination occurs. This process of contamination is stated in Problem (1.2) by the loopback boundary condition $\nu i^{-} I\left(t, i^{-}\right)=\alpha S(t)$, where a zero value of $\alpha$ translates the case without external contamination. It can be seen that such a boundary condition models an intering flux of the disease using the conservation law $\partial_{t} I(t, i)=-\partial_{i}(\nu i I)(t, i)$. Indeed, one then gets for every $t \geq 0$ and $i \in J$,

$$
\frac{d}{d t}\left(\int_{i^{-}}^{i} I(t, i)\right)=\underbrace{\nu i^{-} I\left(t, i^{-}\right)}_{\text {entering flux in } i^{-}} \underbrace{-\nu i I(t, i)}_{\text {entering flux in } i} .
$$

When studying the evolution of a population over time, a fundamental question in ecology is the persistence of the considered population, which consists to know if this last is eternally present or not. In this article, we aim at studying the disease persistence otherwise its exitinction for the infinite dimensional system (1.2). In other words, we wonder if the infected population is persistent or not. This question has been studied in finite dimensional epidemiological systems, see for instance $[8,12,19,21,36,43]$ and all references therein, and in some cases in infinite dimensional systems, see for instance [26, 39, 44] and references therein, and [27, 28, 40,42] for persistence study in the specific case of infection-age models. In the present case, the study strongly depends on the value of the contamination parameter $\alpha$. We prove that, as we could expect by considering a permanent input in the model for the disease, that this last is persistent when the parameter $\alpha$ is positive, and exhibit thresholds on parameters, linked to the basic reproductive number of the disease $\mathcal{R}_{0}$, that ensure persistence or extinction of the disease when $\alpha$ is zero. Moreover, drawing on existing results for the case of age-dependent infectivity SI models, we study 


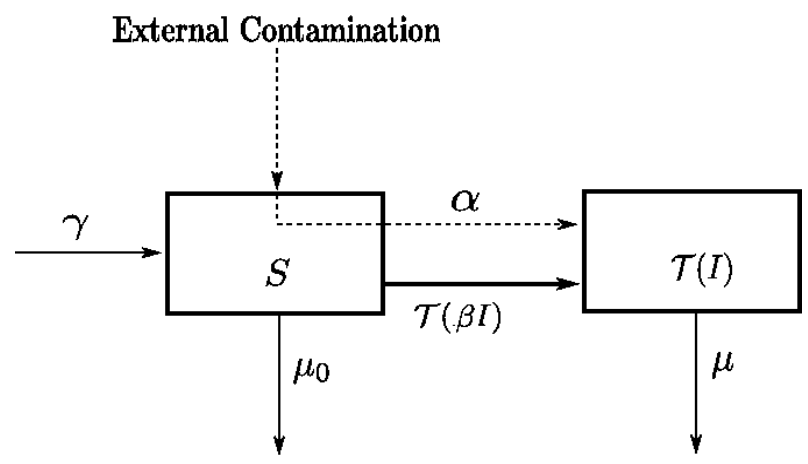

FiG. 1.1. Diagram of population dynamics fluxes

the stability of the disease-free equilibrium and the endemic equilibrium of the system.

This article is structured as follows: we firstly recall in Section 2 some mathematical backgrounds adapted to Problem (1.2): well-posedness, regularity and existence of a semiflow for the problem. In a second step, the Section 3 is dedicated to the mathematical analysis of the time-asymtotic behavior of Problem (1.2). We start this section by recalling some definitions of mathematical epidemiology, that are the notion of epidemic persistence and the concept of $\mathcal{R}_{0}$. Then Section 3 follows with the asymptotic analysis of the model, in both cases with external contamination and without. In the case with contamination, we prove by the use of fluctuation methods that the disease is uniformly stronglypersistent whatever the value of the positive parameter $\alpha$ and exhibit a concrete lower bound for the infective population. In the case without contamination, that is $\alpha=0$, we prove, according to threshold values linked to $\mathcal{R}_{0}$, some asymptotic properties of the problem, such as persistence or extinction of the disease by a study of stability of equilibria of the system. These results are obtained with fluctuation methods and also considering some spectral properties of the differential operator of Problem (1.2). Then Section 4 is devoted to the introduction of a numerical scheme for Problem (1.2) and its numerical analysis. Finally, we apply the results of the previous sections to the transmission of prion diseases in Section 5, where we perform some simulations in specific scenarios (persistence vs. extinction) with concrete parameter values.

2. Well-posedness, regularity and semiflow. In all that follows, $\left(X,\|\cdot\|_{X}\right)$ is the Banach space given by

$$
X=\mathbb{R} \times L^{1}(J),
$$

equiped by the product norm, and $X_{+}$is the non-negative cone of $X$, that is $X_{+}=\mathbb{R}_{+} \times L_{+}^{1}(J)$. For every constant $R>0, B_{R}$ denotes the ball of $X$,

$$
B_{R}=\left\{x \in X,\|x\|_{X} \leq R\right\} .
$$

Linked to Problem (1.2), we consider the differential operator $A: D(A) \subset X \rightarrow X$ defined by

$$
\begin{gathered}
D(A)=\left\{(x, \varphi) \in X,(i \varphi) \in W^{1,1}(J) \text { and } \varphi\left(i^{-}\right)=\alpha x\right\}, \\
A=\left(\begin{array}{cc}
-\mu_{0}-\alpha & 0 \\
0 & L
\end{array}\right),
\end{gathered}
$$

with

$$
L \varphi=-\frac{d}{d i}(\nu i \varphi)-\mu \varphi
$$


and the function $f: X \rightarrow X$ is given by

$$
f(u, v)=\left(\begin{array}{c}
\gamma-u \mathcal{T}(\beta v) \\
\Phi u \mathcal{T}(\beta v)
\end{array}\right)
$$

Then Problem 1.2 rewrites as

$$
\left\{\begin{array}{l}
\frac{d}{d t}\left(\begin{array}{c}
S(t) \\
I(t)
\end{array}\right)=A\left(\begin{array}{c}
S(t) \\
I(t)
\end{array}\right)+f(S(t), I(t)), \\
S(0)=S_{0} \in \mathbb{R}_{+}, \quad I(0, \cdot)=I_{0} \in L_{+}^{1}(J) .
\end{array}\right.
$$

Note the following properties of function $f$ :

Lemma 2.1. The function $f: X \rightarrow X$ given in (2.1) satisfies:

1. $\forall M>0, \forall\left(\left(u_{1}, v_{1}\right),\left(u_{2}, v_{2}\right)\right) \in B_{M}^{2}$,

$$
\left\|f\left(u_{1}, v_{1}\right)-f\left(u_{2}, v_{2}\right)\right\|_{X} \leq 2\|\beta\|_{\infty} M\left\|\left(u_{1}, v_{1}\right)-\left(u_{2}, v_{2}\right)\right\|_{X}
$$

2. $f$ is continuously differentiable and its differential is a compact operator on $X$.

Proof. The first point of the lemma is proved in [33].

From (2.1) one deduces that for $\left(u_{0}, v_{0}\right) \in X$ the differential $D_{\left(u_{0}, v_{0}\right)} f$ of $f$ is given by

$$
D_{\left(u_{0}, v_{0}\right)} f=\left(\begin{array}{ll}
-\mathcal{T}\left(\beta v_{0}\right) & -u_{0} \mathcal{T}(\beta \cdot) \\
\Phi \mathcal{T}\left(\beta v_{0}\right) & \Phi u_{0} \mathcal{T}(\beta \cdot)
\end{array}\right) .
$$

From here, for $(u, v) \in X$ one gets, denoting $\|\cdot\|_{o p}$ the operator norm,

$$
\left\|D_{(u, v)} f-D_{\left(u_{0}, v_{0}\right)} f\right\|_{o p} \leq 2\|\beta\|_{\infty}\left(\left|\mathcal{T}(v)-\mathcal{T}\left(v_{0}\right)\right|+\left|u-u_{0}\right|\right) \leq 2\|\beta\|_{\infty}\left\|(u, v)-\left(u_{0}, v_{0}\right)\right\|_{X},
$$

so $f$ is continuously differentiable from $X$ to $X$ and for every $(u, v) \in X$, the operator $D_{(u, v)} f$ is bounded. Moreover, its range is clearly a finite dimensional space so this operator is compact.

The following theorem, stating the existence and the uniqueness of the solution of Problem (1.2), is proved in [33].

THEOREM 2.2 .

1. The differential operator $(A, D(A))$ is an infinitesimal generator of a strongly continuous positive semigroup $\left\{T_{A}(t)\right\}_{t \geq 0}$ on $X$ that satisfies

$$
\left\|T_{A}(t)\right\| \leq 2 e^{\left(\nu-\mu_{0}\right) t} \quad \forall t \geq 0 .
$$

2. For every $\left(S_{0}, I_{0}\right) \in X_{+}$, Problem (1.2) has a unique mild solution $(S, I) \in C\left(\mathbb{R}_{+}, X_{+}\right)$.

Corollary 2.3. Problem (1.2) induces a continuous semiflow via

$$
\begin{aligned}
\mathbb{R}_{+} \times X_{+} & \rightarrow X_{+} \\
\left(t, x_{0}\right) & \mapsto \phi_{t}\left(x_{0}\right)=(S(t), I(t)),
\end{aligned}
$$

where $(S(t), I(t))$ is the unique solution that satisfies $(S(0), I(0))=x_{0}$.

Proof. For convenience, let us denote $x(t)=(S(t), I(t))$ the unique mild solution of Problem (1.2) with initial condition $x_{0} \in X_{+}$. From the definition of a mild solution, the map $\phi_{t}$ satisfies

$$
\phi_{t}\left(x_{0}\right)=T_{A}(t) x_{0}+\int_{0}^{t} T_{A}(t-s) f\left(\phi_{s}\left(x_{0}\right)\right) d s,
$$

where $\left\{T_{A}(t)\right\}_{t \geq 0}$ is the semigroup of Theorem 2.2 and function $f$ is defined in (2.1).

Then the semigroup property of $\left\{T_{A}(t)\right\}_{t \geq 0}$ clearly implies that for every $\tau \geq 0$,

$$
\phi_{t} \circ \phi_{\tau}\left(x_{0}\right)=\phi_{t+\tau}\left(x_{0}\right),
$$


and so $\left(t, x_{0}\right) \mapsto \phi_{t}\left(x_{0}\right)$ is a semiflow on $\mathbb{R}_{+} \times X_{+}$.

We now prove the continuity. Let $M>0$ and $\left(\tau, x_{0}\right),\left(t, y_{0}\right) \in B_{M}$. Then, from Lemma 2.1 and equation $(2.3)$, one gets

$$
\left\|\phi_{\tau}\left(x_{0}\right)-\phi_{t}\left(y_{0}\right)\right\|_{X} \leq\left\|\phi_{\tau}\left(x_{0}\right)-\phi_{t}\left(x_{0}\right)\right\|_{X}+2 e^{\left(\nu-\mu_{0}\right) t}\left(1+2 M t\|\beta\|_{\infty}\right)\left\|x_{0}-y_{0}\right\|_{X}
$$

A consequence of Theorem 2.2 is that $t \mapsto \phi_{t}\left(x_{0}\right) \in C\left(\mathbb{R}_{+}, X_{+}\right)$and so the latter inequality proves the continuity of the semiflow.

Since from Lemma 2.1 function $f$ is continuously differentiable, the following corollary is a consequence of the regularity theorem p.187 in [30] :

Corollary 2.4. Suppose that the initial condition satisfies $\left(S_{0}, I_{0}\right) \in D(A)$. Then the mild solution of Problem (1.2) is a classical solution.

3. Asymptotic properties. We assume in all that follows that the initial condition $\left(S_{0}, I_{0}\right) \in$ $D(A) \cap X_{+}$and is not zero.

3.1. Definitions. In this section, we express some standard notions from mathematical ecology by formulating in the context of Problem (1.2) the definitions of $\mathcal{R}_{0}$ (see [1,13] for an introduction) and persistence (see $[7,17,43])$.

In epidemiology, a fundamental concept is the basic reproduction number of the disease, denoted $\mathcal{R}_{0}$, i.e. the number of secondary infections resulting from a single primary infection into an otherwise susceptible population. Mathematically, $\mathcal{R}_{0}$ is looked as a threshold, depending on epidemiological parameters of the problem, that ensures or not the stability of an equilibrium point called disease-free equilibrium when this latter exists.

Another fundamental epidemiological concept linked to $\mathcal{R}_{0}$ is the disease persistence. Let $\rho: X \rightarrow \mathbb{R}_{+}$ be a nonnegative uniformly continuous function on $X$ and consider the composition $\sigma_{\rho}(t, x)=\rho\left(\phi_{t}(x)\right)$, where $\phi_{t}$ is the semiflow defined in Corollary 2.3. Remark that this latter corollary implies that $\sigma_{\rho}$ is a continuous map from $\mathbb{R}_{+} \times X_{+}$to $\mathbb{R}_{+}$. Let us introduce the following notations, that will be used in all that follows :

$$
\sigma_{\rho}^{+}(x)=\limsup _{t \rightarrow+\infty} \sigma_{\rho}(t, x), \quad \sigma_{\rho}^{-}(x)=\liminf _{t \rightarrow+\infty} \sigma_{\rho}(t, x)
$$

REMARK 1. If no misunderstanding about the initial condition $x \in X$ is possible, we may omit the $x$-dependence by writing $\sigma_{\rho}(t)$ rather than $\sigma_{\rho}(t, x)$ and $\sigma_{\rho}^{ \pm}$rather than $\sigma_{\rho}^{ \pm}(x)$.

Here is the definition of persistence for our system.

DEFinition 3.1. The semiflow $\phi_{t}$ is said

- strongly $\rho$-persistent if

$$
\forall x \in X, \quad \rho(x)>0 \Rightarrow \sigma_{\rho}^{-}(x)>0
$$

- uniformly strongly $\rho$-persistent if there exists $\varepsilon>0$ such that

$$
\forall x \in X, \quad \rho(x)>0 \Rightarrow \sigma_{\rho}^{-}(x) \geq \varepsilon
$$

The semiflow $\phi_{t}$ is said weakly $\rho$-persistent, respectively uniformly weakly $\rho$-persistent, if the considered limits above are $\sigma_{\rho}^{+}(x)$ instead of $\sigma_{\rho}^{-}(x)$.

With the above definition, we say that:

- the total population in Problem (1.2) is persistent if the semiflow $\phi_{t}$ is $\tilde{\rho}$-persistent for the mapping $\tilde{\rho}:(u, v) \in X \mapsto u+\mathcal{T}(v)$; 
- the disease in Problem (1.2) is persistent if the semiflow $\phi_{t}$ is $\rho$-persistent for the mapping $\rho:(u, v) \in X \mapsto \mathcal{T}(v)$.

One can now give a necessary condition to get persistence of the disease.

Lemma 3.2. The total population in Problem (1.2) is a non negative bounded function which is uniformly strongly persistent if $\gamma>0$ and converges to 0 if $\gamma=0$. Moreover, for $\gamma>0$ the set $X_{0}=\{x \in$ $\left.X_{+}, \tilde{\rho}(x) \leq \frac{\gamma}{\mu_{0}}\right\}$ is an invariant set for the system (1.2).

Proof. The quantity $\sigma_{\tilde{\rho}}\left(t,\left(S_{0}, I_{0}\right)\right)$ clearly represents the total population in Problem (1.2). The positivity of the solution stated by Theorem 2.2 directly yields the positivity of the total population for initial densities such that $\tilde{\rho}\left(S_{0}, I_{0}\right) \geq 0$.

Applying the operator $\mathcal{T}$ to the transport equation in (1.2) one deduces, using the boundary condition, assumption (ii) on $\Phi$ and equation (3.5), the equality

$$
\sigma_{\tilde{\rho}}^{\prime}(t)=\gamma-\mu_{0} S(t)-\mathcal{T}(\mu I)(t) .
$$

Since $\mu_{0} \leq \mu(i) \leq\|\mu\|_{\infty}$ for a.e. $i \in J$, the latter equality implies that the total population $\sigma_{\tilde{\rho}}(t)$ satisfies the differential inequalities

$$
\gamma-\|\mu\|_{\infty} \sigma_{\tilde{\rho}}(t) \leq \sigma_{\tilde{\rho}}^{\prime}(t) \leq \gamma-\mu_{0} \sigma_{\tilde{\rho}}(t) .
$$

Then using standard Gronwall arguments one deduces that

$$
f_{\infty}(t) \leq \sigma_{\tilde{\rho}}(t) \leq f_{0}(t)
$$

where $f_{0}$ is the following function,

$$
f_{0}(t)=e^{-\mu_{0} t} \tilde{\rho}\left(S_{0}, I_{0}\right)+\frac{\gamma}{\mu_{0}}\left(1-e^{-\mu_{0} t}\right),
$$

and $f_{\infty}(t)$ is the same function as $f_{0}$ but with $\|\mu\|_{\infty}$ instead of $\mu_{0}$. It follows from (3.2) that the total population is a bounded function. Moreover, letting $t \rightarrow+\infty$ in (3.2) one gets

$$
\frac{\gamma}{\|\mu\|_{\infty}} \leq \sigma_{\tilde{\rho}}^{-} \leq \sigma_{\tilde{\rho}}^{+} \leq \frac{\gamma}{\mu_{0}}
$$

which proves the persistence result.

Finally, from (3.2) one deduces that for $\gamma>0, \sigma_{\tilde{\rho}}(t, x) \leq \frac{\gamma}{\mu_{0}}$ for every $x \in X_{0}$, that is equivalent to $\phi_{t}\left(X_{0}\right) \subset X_{0}$, which ends the proof of the lemma.

As a consequence of Lemma 3.2, we focus on the study in the case where the total population does not extinct by assuming in all that follows that $\gamma>0$.

3.2. Study in a particular case. We now look at the particular case where $\mu$ and $\beta$ are non negative constant parameters. We therefore suppose in all that follows the following assumption,

$$
\mu(i):=\mu_{1} \geq \mu_{0}, \text { and } \beta(i):=\beta_{0} \text { for a.e. } i \in J .
$$

Then denoting $Y(t)=\mathcal{T}(I)(t)$ one gets the following proposition.

Proposition 3.3. Suppose that assumption $(H)$ is satisfied. Then $(S(t), Y(t))$ satisfies for every $t \geq 0$ the following differential equations system,

$$
\left\{\begin{array}{l}
\frac{d S(t)}{d t}=\gamma-\left(\mu_{0}+\alpha\right) S(t)-\beta_{0} S(t) Y(t) \\
\frac{Y(t)}{d t}=\alpha S(t)+\beta_{0} S(t) Y(t)-\mu_{1} Y(t) \\
S(0)=S_{0}, \quad Y(0)=\mathcal{T}\left(I_{0}\right)
\end{array}\right.
$$


Proof. Let us start by proving that the infected stage satisfies the following asymptotic property,

$$
\lim _{i \rightarrow+\infty} i I(t, i)=0 .
$$

Indeed from Corollary 2.4 one gets $i I(t, i) \in W^{1,1}(J)$ for every $t \geq 0$. Then from the equality

$$
i I(t, i)=\int_{i^{-}}^{i} \partial_{u}(u I(t, u)) d u+\frac{\alpha}{\nu} S(t),
$$

one deduces that for every $t \geq 0$ there exists $l(t) \geq 0$ such that $\lim _{i \rightarrow+\infty} i I(t, i)=l(t)$. Finally, since $i I(t, i) \in W^{1,1}(J) \subset L^{1}(J)$, then necessarily $l(t)=0$ for every $t \geq 0$ and $(3.5)$ is then proved.

Integrating w.r.t. $i \in J$ the transport equation of Problem (1.2) yields, as a consequence of the expression of the boundary condition and of the limit stated by (3.5),

$$
\begin{aligned}
\frac{d Y(t)}{d t} & =\nu i^{-} I\left(t, i^{-}\right)+\beta_{0} S(t) Y(t)-\mu_{1} Y(t) \\
& =\alpha S(t)+\beta_{0} S(t) Y(t)-\mu_{1} Y(t),
\end{aligned}
$$

and so the proposition is proved.

REMARK 2. Observe that from (3.4), we obtain the following balance equation

$$
\frac{d}{d t}[S(t)+Y(t)]=\gamma-\mu_{0} S(t)+\mu_{1} Y(t),
$$

which states that the total population can change only due to the entering flux and death. Note that, using the mapping $\rho$ and $\tilde{\rho}$ previously defined, this equation can also be written as

$$
\frac{d}{d t}\left[\sigma_{\tilde{\rho}}(t)\right]=\gamma-\mu_{0} \sigma_{\tilde{\rho}}(t)+\left(\mu_{0}+\mu_{1}\right) \sigma_{\rho}(t),
$$

where the initial condition $\left(S_{0}, I_{0}\right)$ is deliberately avoided.

In the particular case where assumption $(H)$ is satisfied, we consider the $\mathcal{R}_{0}$ value given by

$$
\mathcal{R}_{0}=\frac{\gamma \beta_{0}}{\mu_{0} \mu_{1}} .
$$

Proposition 3.4. Suppose that assumption $(H)$ is satisfied. Then

- If $\alpha>0$, Problem (3.4) has a unique equilibrium point $(\bar{S}, \bar{Y})$ given by

$$
\bar{Y}=\frac{\delta+\sqrt{\delta^{2}+4 \alpha \gamma \beta_{0} \mu_{1}}}{2 \beta \mu_{1}}, \quad \bar{S}=\frac{\mu_{1} \bar{Y}}{\alpha+\beta_{0} \bar{Y}}
$$

with $\delta=\gamma \beta_{0}-\left(\mu_{0}+\alpha\right)$.

- If $\alpha=0$, then

- $\quad \mathcal{R}_{0} \leq 1 \Rightarrow$ there exists a unique equilibrium $E_{0}=\left(\frac{\gamma}{\mu_{0}}, 0\right)$,

- $\quad \mathcal{R}_{0}>1 \Rightarrow$ there are two equilibria, $E_{0}$ and $E_{*}=\left(\frac{\mu_{1}}{\beta_{0}}, \frac{\mu_{0}}{\beta_{0}}\left(\mathcal{R}_{0}-1\right)\right)$

Proof. Suppose that $\alpha>0$. Looking for a steady state $(\bar{S}, \bar{Y})$ in (3.4), the second equation gives the equality that links $\bar{S}$ to $\bar{Y}$ in (3.7). From here, substituting the $\bar{S}$ value in the first equation one gets

$$
\beta_{0} \mu_{1} \bar{Y}^{2}-\delta \bar{Y}-\alpha \gamma=0 .
$$


The latter equation has only one admissible point that is the non negative value $\bar{Y}$ stated in (3.7). Suppose now that $\alpha=0$. Then for $\bar{Y}=0$ one gets $\bar{S}=\frac{\gamma}{\mu_{0}}$ from the first equation leading to the equilibrium $E_{0}$. Moreover, taking $\alpha=0$ in (3.7) yields $\bar{S}=\frac{\mu_{1}}{\beta_{0}}$ and then the first equation in (3.4) implies that $\gamma-\frac{\mu_{0} \mu_{1}}{\beta_{0}}-\mu_{1} \bar{Y}=0$ and so one deduces $E_{*}$ as given in the proposition.

In the case where assumption $(H)$ is satisfied and $\alpha>0$, the latter proposition implies that the disease may not extinct. The following theorem is a particular result of a more general result that will proved without the assumption $(H)$ (see Theorem 3.11 and 3.12).

TheOREm 3.5. Suppose that assumption $(H)$ is satisfied and $I_{0}>0$. If $\alpha>0$ then the disease is uniformly strongly persistent. If $\alpha=0$ then

$\mathcal{R}_{0}<1 \Rightarrow E_{0}$ is locally asymptotically stable,

$\mathcal{R}_{0}>1 \Rightarrow E_{*}$ is locally asymptotically stable and the disease is uniformly strongly persistent.

3.3. Mathematical study in the general case. We now suppose that assumption $(H)$ is not satisfied, translating that the mortality rate and the transmission rate are infection-load dependent functional parameters satisfying hypothesis (iii) .

3.3.1. Disease with external contamination. This case is translated by the assumption $\alpha>0$.

THEOREM 3.6. Suppose that $I_{0}>0$ and $\alpha>0$. Then the disease is uniformly strongly persistent, and we have the following lower bound,

$$
\sigma_{\rho}^{-}\left(S_{0}, I_{0}\right) \geq \frac{\alpha \gamma \mu_{0}}{\|\mu\|_{\infty}\left(\mu_{0}^{2}+\alpha \mu_{0}+\|\beta\|_{\infty} \gamma\right)} .
$$

Proof. To prove this theorem, we make several uses of fluctuation method (see [20, 43]). Let us denote in all the proof the following limit,

$$
S^{-}=\liminf _{t \rightarrow+\infty} S(t)
$$

By fluctuation method, there exists a sequence $t_{n} \rightarrow+\infty$ such that

$$
\lim _{n \rightarrow+\infty} S\left(t_{n}\right)=S^{-}, \quad \lim _{n \rightarrow+\infty} S^{\prime}\left(t_{n}\right)=0 .
$$

Since $\gamma>0$, Lemma 3.2 implies that $t \mapsto \tilde{\rho}(t)$ is bounded and so $\sigma_{\rho}^{+}<+\infty$ and same for $S^{+}$.

Let $\varepsilon>0$. By definition of $\sigma_{\rho}^{+}$one gets $\lim \sup _{n \rightarrow+\infty} \sigma_{\rho}\left(t_{n}\right) \leq \sigma_{\rho}^{+}<\sigma_{\rho}^{+}+\varepsilon$ and so $\sigma_{\rho}\left(t_{n}\right) \leq \sigma_{\rho}^{+}+\varepsilon$ for every $n$ big enough. The differential equation of susceptibles in Problem (1.2) then gives for every $n$ big enough,

$$
S^{\prime}\left(t_{n}\right) \geq \gamma-\left(\mu_{0}+\alpha\right) S\left(t_{n}\right)-\|\beta\|_{\infty} S\left(t_{n}\right)\left(\sigma_{\rho}^{+}+\varepsilon\right) .
$$

From the latter equation, for $n \rightarrow \infty$ one gets

$$
S^{-} \geq \frac{\gamma}{\mu_{0}+\alpha+\|\beta\|_{\infty}\left(\sigma_{\rho}^{+}+\varepsilon\right)}>0 .
$$

Furthermore, the fluctuation method also implies that there exists a sequence $s_{n} \rightarrow+\infty$ such that

$$
\begin{aligned}
& \lim _{n \rightarrow+\infty} \sigma_{\rho}\left(s_{n}\right)=\sigma_{\rho}^{-} \\
& \lim _{n \rightarrow+\infty} \sigma_{\rho}^{\prime}\left(s_{n}\right)=0 .
\end{aligned}
$$


Since $S^{-}>0$, one can consider now $0<\varepsilon^{\prime}<S^{-}$. Then one gets $S\left(s_{n}\right)>S^{-}-\varepsilon^{\prime}$ for every $n$ big enough. The inequality $\mu(i) \geq\|\mu\|_{\infty}$ f.a.e. $i \in J$ and equation (3.1) imply that for every $n$ big enough,

$$
\sigma_{\rho}^{\prime}\left(s_{n}\right) \geq \alpha\left(S^{-}-\varepsilon^{\prime}\right)-\|\mu\|_{\infty} \sigma_{\rho}\left(s_{n}\right) .
$$

For $n \rightarrow \infty$ one finally gets, taking (3.9) into account,

$$
\sigma_{\rho}^{-} \geq \frac{\alpha}{\|\mu\|_{\infty}}\left(\frac{\gamma}{\mu_{0}+\alpha+\|\beta\|_{\infty}\left(\sigma_{\rho}^{+}+\varepsilon\right)}-\varepsilon^{\prime}\right) .
$$

Now, letting $\varepsilon, \varepsilon^{\prime} \rightarrow 0$,

$$
\sigma_{\rho}^{-} \geq \frac{\alpha \gamma}{\|\mu\|_{\infty}\left(\mu_{0}+\alpha+\|\beta\|_{\infty} \sigma_{\rho}^{+}\right)} .
$$

Moreover, equation (3.3) induces that $\sigma_{\rho}^{+} \leq \frac{\gamma}{\mu_{0}}$ so the latter bound is independent of the initial condition, and the disease is uniformly persistent and the lower bound in the theorem is satisfied.

3.3.2. Disease without external contamination. We now study the case without external contamination, that is $\alpha=0$. To perform the persistence analysis, we exhibit some spectral properties of the differential operator $A$ and of the semigroup $\left\{T_{A}(t)\right\}_{t \geq 0}$ of Theorem 2.2.

Before proceeding, we introduce some notations and recall some definitions of spectral theory.

Denoting $\rho(A)$ the resolvent set of $A: D(A) \subset X \rightarrow X$, we define the spectrum $\sigma(A)$, the point spectrum $\sigma_{p}(A)$ and the spectral bound $s(A)$ by

$$
\begin{gathered}
\sigma(A)=\mathbb{C} \backslash \rho(A), \\
\sigma_{p}(A)=\{\lambda \in \mathbb{C}, \lambda I-A \text { is not injective }\}, \\
s(A)=\sup \{\operatorname{Re} \lambda, \lambda \in \sigma(A)\} .
\end{gathered}
$$

For an isolated $\lambda_{0} \in \sigma(A)$, the associated spectral projection $P_{\lambda_{0}}$ is

$$
P_{\lambda_{0}}=\frac{1}{2 \pi i} \int_{\gamma_{\lambda_{0}}} R_{\lambda} d \lambda
$$

where $R_{\lambda}$ is the resolvent operator of $(A, D(A))$ and $\gamma_{\lambda_{0}}$ is a Jordan path in the complement of $\sigma(A) \backslash\left\{\lambda_{0}\right\}$ enclosing $\left\{\lambda_{0}\right\}$.

The algebraic multiplicity $m_{a}\left(\lambda_{0}\right) \in \mathbb{N} \cup\{+\infty\}$ of $\lambda_{0}$ is the dimension of the range of $P_{\lambda_{0}}$. We recall the following fundamental result of spectral theory (see Yosida [49]) :

Theorem 3.7. If $m_{a}\left(\lambda_{0}\right)<\infty$ then $\lambda_{0} \in \sigma_{p}(A)$.

Denoting $\mathcal{L}(X)$ the set of bounded linear operators on $X$ and $\mathcal{K}(X)$ the subset of compact operators on $X$, we then define the essential norm $\|L\|_{\text {ess }}$ of $L \in \mathcal{L}(X)$ by

$$
\|L\|_{\text {ess }}=\inf _{K \in \mathcal{K}(X)}\|L-K\|_{X}
$$

We recall that the quotient $\mathcal{L}(X) / \mathcal{K}(X)$ is called the Calkin algebra which, when providing the norm

$$
\|\hat{L}\|=\|L\|_{\mathrm{ess}}
$$

where $\hat{L}=L+\mathcal{K}(X)$, is a Banach algebra with unit (see [15] and references cited in for details). The growth bound $\omega_{0}(A) \in[-\infty,+\infty)$ of $A$ is defined by

$$
\omega_{0}(A)=\lim _{t \rightarrow+\infty} \frac{1}{t} \ln \left(\left\|T_{A}(t)\right\|_{X}\right),
$$


and the essential growth bound $\omega_{\mathrm{ess}}(A) \in[-\infty,+\infty)$ of $A$ is

$$
\omega_{\mathrm{ess}}(A)=\lim _{t \rightarrow+\infty} \frac{1}{t} \ln \left(\left\|T_{A}(t)\right\|_{\mathrm{ess}}\right) .
$$

The following theorem that gives a characterization of the growth bound using the spectrum of $A$ was proved by Engel and Nagel [15].

THEOREM 3.8. The growth bound of A satisfies

$$
\omega_{0}(A)=\max \left\{\omega_{\mathrm{ess}}(A), s(A)\right\},
$$

and for every $\omega>\omega_{\mathrm{ess}}(A)$ the set $\sigma_{\omega}=\{\lambda \in \sigma(A), \operatorname{Re}(\lambda)>\omega\}$ is finite and composed of finite algebraic multiplicity elements.

A consequence of Theorems 3.7 and 3.8 is that

$$
\omega>\omega_{\mathrm{ess}}(A) \Rightarrow \sigma_{\omega} \subset \sigma_{p}(A) .
$$

REMARK 3. Another definition of the essential norm can be stated by the use of the Kuratovski measure of non-compactness ([46, 47]). However, this definition is equivalent to the one we use (see [3]). Due to the quotient defined previously, the use of the Calkin algebra shows well why the compact operators do not affect the growth bound values. More specifically, one gets $\omega_{\mathrm{ess}}(A+K)=\omega_{\mathrm{ess}}(A)$ for every $K \in \mathcal{K}(X)$.

In all that follows, let us consider for every $\mu \in L^{\infty}(J)$ the function $\pi_{\mu} \in L^{1}(J)$, and the values $\mathcal{R}_{-}$, $\mathcal{R}_{0}$ and $\mathcal{R}_{+}$given by

$$
\begin{gathered}
\pi_{\mu}(i)=\frac{1}{\nu i} \int_{i^{-}}^{i} \Phi(s) e^{-\int_{s}^{i} \frac{\mu(l)}{\nu l} d l} d s \\
\mathcal{R}_{-}=\frac{\gamma \beta_{0}}{\mu_{0}\|\mu\|_{\infty}}, \quad \mathcal{R}_{0}=\frac{\gamma}{\mu_{0}} \mathcal{T}\left(\beta \pi_{\mu}\right), \quad \mathcal{R}_{+}=\frac{\gamma\|\beta\|_{\infty}}{\mu_{0}^{2}} .
\end{gathered}
$$

REMARK 4. One can check that the following equalities are satisfied,

$$
\mathcal{R}_{-}=\frac{\gamma}{\mu_{0}} \mathcal{T}\left(\beta_{0} \pi_{\|\mu\|_{\infty}}\right), \quad \mathcal{R}_{+}=\frac{\gamma}{\mu_{0}} \mathcal{T}\left(\|\beta\|_{\infty} \pi_{\mu_{0}}\right),
$$

and so the three values $\mathcal{R}_{-}, \mathcal{R}_{0}$ and $\mathcal{R}_{+}$are related by the inequalities $\mathcal{R}_{-} \leq \mathcal{R}_{0} \leq \mathcal{R}_{+}$. As a consequence, one gets $\mathcal{R}_{-}>1 \Rightarrow \mathcal{R}_{0}>1$ and $\mathcal{R}_{+}<1 \Rightarrow \mathcal{R}_{0}<1$.

Proposition 3.9.

- If $\mathcal{R}_{0} \leq 1$ then Problem (1.2) has a unique equilibrium $E_{0} \in X_{+}$, that is the disease free equilibrium given by

$$
E_{0}=\left(S_{F}, 0\right)=\left(\frac{\gamma}{\mu_{0}}, 0\right)
$$

- If $\mathcal{R}_{0}>1$ then Problem (1.2) has two equilibria in $X_{+}$, that are the disease free equilibrium $E_{0}$ and an endemic equilibrium $E_{*}$ given by

$$
E_{*}=\left(S_{*}, I_{*}\right)=\left(\frac{S_{F}}{\mathcal{R}_{0}}, \frac{\gamma\left(\mathcal{R}_{0}-1\right)}{\mathcal{R}_{0}} \pi_{\mu}\right)
$$

Proof. Looking for $(\bar{S}, \bar{I}) \in X_{+}$constant solutions of Problem (1.2) one gets

$$
\left\{\begin{array}{l}
0=\gamma-\mu_{0} \bar{S}-\bar{S} \mathcal{T}(\beta \bar{I}), \\
(\nu i \bar{I})^{\prime}=-\mu(i) \bar{I}(i)+\Phi(i) \bar{S} \mathcal{T}(\beta \bar{I}), \quad i \in J \\
\nu i^{-} I\left(i^{-}\right)=0 .
\end{array}\right.
$$


The first equation and the integration of the second equation give

$$
\begin{gathered}
\bar{S}=\frac{\gamma}{\mu_{0}+\mathcal{T}(\beta \bar{I})}, \\
\bar{I}(i)=\bar{S} \mathcal{T}(\beta \bar{I}) \pi_{\mu}(i) .
\end{gathered}
$$

Applying the $\mathcal{T}(\beta \cdot)$-operator to (3.12) yields

$$
\mathcal{T}(\beta \bar{I})\left(1-\frac{\bar{S} \mathcal{R}_{0}}{S_{F}}\right)=0
$$

From here, either $\mathcal{T}(\bar{I})=0$, and since $\mathcal{T}(\bar{I}) \leq \frac{\mathcal{T}(\beta \bar{I})}{\beta_{0}}$ so $\bar{I}=0$ in $L^{1}(J)$ and (3.11) implies that $E_{0}$ is the desired disease free equilibrium, or $\mathcal{T}(\bar{I})>0$. In that case $\bar{I}$ is not zero in $L^{1}(J)$. Then necessarily $\bar{S}=S_{*}$ and from (3.11) follows

$$
\mathcal{T}(\beta \bar{I})=\left(\frac{\gamma}{S_{*}}-\mu_{0}\right)=\mu_{0}\left(\mathcal{R}_{0}-1\right)
$$

This latter is positive if and only if $\mathcal{R}_{0}>1$, and then (3.12) finally implies

$$
\bar{I}=S_{*} \mathcal{T}(\beta \bar{I}) \pi_{\mu}=I_{*} .
$$

प

Since the semilinear part $f$ defined in (2.1) is continuously differentiable, a result from the theory of semilinear evolution equations proves that, under some conditions on the growth bound $\omega_{0}(A)$ and on the essential growth bound $\omega_{\text {ess }}(A)$, it is possible to approach the stability of some equilibrium points for the infinite-dimensional system (1.2) in the same way as for a finite dimensional system. More precisely, the following proposition hods:

Proposition 3.10. For every equilibrium point $E$ of the Problem (2.2), we have

1. $\omega_{0}\left(A+D_{E} f\right)<0$ implies that $E$ is locally asymptotically stable.

2. $\omega_{0}\left(A+D_{E} f\right)>0$ and $\omega_{\mathrm{ess}}\left(A+D_{E} f\right) \leq 0$ implies instability of $E$.

We refer to [35] or Proposition 4.19 in [46] for the proof of this result.

Using this result one gets the following theorem proving that the disease may extinct when $\mathcal{R}_{0}<1$ whereas the disease-free equilibrium is unstable when $\mathcal{R}_{0}>1$. Moreover, we exhibit stronger assumptions on $\mathcal{R}_{-}$and $\mathcal{R}_{+}$that provide either the global stability of the disease-free equilibrium, either the uniform strong persistence of the disease.

TheOREM 3.11. Suppose that $I_{0}>0$ and $\mathcal{R}_{0}<1$. Then the disease free equilibrium $E_{0}$ is locally asymptotically stable. Moreover, under the stronger assumption $\mathcal{R}_{+}<1$, the disease free equilibrium $E_{0}$ is globally asymptotically stable.

Proof. The linearized system (1.2) about the disease-free equilibrium $E_{0}$ is

$$
\frac{d}{d t}\left(\begin{array}{c}
S(t) \\
I(t)
\end{array}\right)=\left(A+D_{E_{0}} f\right)\left(\begin{array}{c}
S(t) \\
I(t)
\end{array}\right)
$$

where $D_{E_{0}} f$ is the differential at the disease free equilibrium. We first prove that $\omega_{0}\left(A+D_{E_{0}} f\right)<0$. The estimation $(2.3)$ shows that $\omega_{0}(A) \leq \nu-\mu_{0}$, so does not guarantee a negative value of the growth bound when the exponential velocity $\nu$ is too big. However, one can prove that $\omega_{\text {ess }}(A)<0$. Indeed the change of variables using the mapping $(t, \xi) \mapsto(t, i)=\left(t, i^{-} e^{\nu(t-\xi)}\right)$ and standard integrative computations imply that the semigroup $T_{A}(t)$ satisfies for every $t \geq 0$ and every $(u, v) \in X$,

$$
T_{A}(t)\left(\begin{array}{c}
u \\
v
\end{array}\right)=v\left(i e^{-\nu t}\right)\left(\begin{array}{c}
0 \\
e^{-\int_{0}^{t}\left(\mu\left(i e^{-\nu s}\right)+\nu\right) d s} \chi_{\{\theta(i) \geq t\}}
\end{array}\right)+u\left(\begin{array}{c}
e^{-\mu_{0} t} \\
0
\end{array}\right),
$$


where $\theta(i)=\frac{1}{\nu} \ln \left(\frac{i}{i^{-}}\right)$. Moreover, a change of variables clearly gives

$$
\int_{\{\theta \leq t\}} v\left(i e^{-\nu t}\right) d s \leq e^{\nu t}\|v\|_{L^{1}(J)},
$$

and since the $u$-part in (3.14) has rank 1, then it is compact and one gets with assumption (iii) on $\mu$,

$$
\left\|T_{A}(t)\right\|_{\text {ess }} \leq e^{-\mu_{0} t} .
$$

From here, $\omega_{\text {ess }}(A) \leq-\mu_{0}$, and since from Lemma 2.1.2 the differential $D_{E_{0}} f$ is a compact operator, one deduces that $\omega_{\text {ess }}\left(A+D_{E_{0}} f\right)<0$. As a consequence of Theorems 3.7 and 3.8 we only have to focus on the point spectrum of $A+D_{E_{0}} f$, by proving that $\sup \left\{\operatorname{Re} \lambda, \lambda \in \sigma_{p}\left(A+D_{E_{0}} f\right)\right\}<0$ when $\mathcal{R}_{0}<1$.

For $\lambda \in \mathbb{C}$ such that $\operatorname{Re} \lambda>-\mu_{0}$ and looking for a solution $I(t, i)=z(i) e^{\lambda t}$ of the linearized problem (3.13), function $z$ satisfies

$$
\lambda z(i)+(i \nu z(i))^{\prime}=-\mu(i) z(i)+\frac{\gamma}{\mu_{0}} \Phi(i) \mathcal{T}(\beta z) .
$$

Since the boundary is homogeneous, an integration then gives

$$
z(i)=\frac{\gamma}{\mu_{0}} \mathcal{T}(\beta z) \pi_{\mu+\lambda}
$$

and applying the operator $\mathcal{T}(\beta \cdot)$ leads to the following characteristic equation for $\lambda \in \mathbb{C}, \operatorname{Re} \lambda>-\mu_{0}$,

$$
\xi(\lambda)=1
$$

where $\xi(\lambda)=\frac{\gamma}{\mu_{0}} \mathcal{T}\left(\beta \pi_{\mu+\lambda}\right)$. Suppose now that $\mathcal{R}_{0}<1$. On can check that $\xi(0)=\mathcal{R}_{0}<1$. Moreover, for $\lambda \in \mathbb{R}$ the Lebesgue dominated convergence theorem implies that $\lambda \mapsto \xi(\lambda)$ is a strictly decreasing function on $\left(-\mu_{0}, \infty\right)$ and

$$
\lim _{\lambda \rightarrow+\infty} \xi(\lambda)=0 .
$$

Then necessarily (3.15) implies that $\lambda \in\left(-\mu_{0}, 0\right)$ and $\sup \left\{\sigma_{p}\left(A+D_{E_{0}} f\right) \cap \mathbb{R}\right\}<0$. Now, for $\lambda \in \mathbb{C}$ such that $\operatorname{Re} \lambda>-\mu_{0}$ one gets, considering the real part in (3.15),

$$
1=\frac{\gamma}{\mu_{0}} \mathcal{T}\left(\frac{\beta(i)}{\nu i} \int_{i^{-}}^{i} \Phi(s) \cos (\operatorname{Im} \lambda \ln (i / s)) e^{-\int_{s}^{i} \frac{\operatorname{Re} \lambda+\mu(l)}{\nu l} d l} d s\right) \leq \xi(\operatorname{Re} \lambda) .
$$

From here, $\mathcal{R}_{0}<1 \Rightarrow \operatorname{Re} \lambda<0$. To conclude, $A+D_{E_{0}} f$ has a dominant eigenvalue with negative real part for $\mathcal{R}_{0}<1$ so Theorem 3.8 implies that $\omega_{0}\left(A+D_{E_{0}} f\right)<0$. Proposition 4.19 in [46] finally gives the stability of the disease-free equilibrium $E_{0}$.

We now suppose that $\mathcal{R}_{+}<1$ and prove the global stability of the disease free equilibrium using the fluctuation method. The goal is to prove that $\sigma_{\rho}^{+}=0$, where $\rho:(u, v) \in X \mapsto \mathcal{T}(v)$.

Applying the operator $\mathcal{T}$ to the infected equation in Problem (1.2), one deduces from assumption (iii) on parameter $\mu$ that

$$
\frac{d \mathcal{T}(I)}{d t} \leq \alpha S+\|\beta\|_{\infty} S \mathcal{T}(I)-\mu_{0} \mathcal{T}(I)
$$

Then the fluctuation method implies that

$$
\sigma_{\rho}^{+}\left(\|\beta\|_{\infty} S^{+}-\mu_{0}\right) \geq 0
$$


where

$$
S^{+}=\limsup _{t \rightarrow+\infty} S(t)
$$

From (3.2) one deduces that

$$
\frac{\gamma}{\mu_{0}} \geq \sigma_{\tilde{\rho}}^{+} \geq S^{+}
$$

so one gets

$$
\sigma_{\rho}^{+}\left(\mathcal{R}_{+}-1\right) \geq 0
$$

which proves that, unformly in $\left(S_{0}, I_{0}\right), \sigma_{\rho}^{+}=0$ when $\mathcal{R}_{+}<1$. Then the global stability of $E_{0}$ yields.

TheOREM 3.12. Suppose that $I_{0}>0$ and $\mathcal{R}_{0}>1$. Then the disease free equilibrium $E_{0}$ is unstable and the endemic equilibrium $E_{*}$ is locally asymptotically stable. Moreover, under the stronger assumption $\mathcal{R}_{-}>1$, the disease is uniformly strongly persistent.

Proof. Same calculus than in the proof of Theorem 3.11 proves that for $\mathcal{R}_{0}>1$ the disease free equilibrium $E_{0}$ is unstable. Indeed, since $\xi(0)=\mathcal{R}_{0}>1$, continuity and decreasing of $\lambda \mapsto \xi(\lambda)$ on $\mathbb{R}$ imply that there exists an unique $\lambda_{0}>0$ such that $\xi\left(\lambda_{0}\right)=1$. So $\omega_{0}\left(A+D_{E_{0}} f\right)>0$ and the unstability yields from Proposition 3.10.

We now prove on the stability of the endemic equilibrium $E_{*}$. Since $\omega_{\text {ess }}\left(A+D_{E_{*}} f\right)<0$, we only have to focus on the point spectrum of $A+D_{E_{*}} f$. The linearized system (1.2) about $E_{*}$ rewrites, using the expression of equilibria $E_{0}, E_{*}$ and $\mathcal{R}_{0}$,

$$
\left\{\begin{array}{l}
\frac{d S(t)}{d t}=-\mu_{0} \mathcal{R}_{0} S(t)-\frac{S_{F}}{\mathcal{R}_{0}} \mathcal{T}(\beta I)(t), \quad t \geq 0 \\
\frac{\partial I(t, i)}{\partial t}=-\frac{\partial(\nu i I)(t, i)}{\partial i}-\mu(i) I(t, i)+\Phi(i) \frac{S_{F}}{\mathcal{R}_{0}} \mathcal{T}(\beta I)(t)+\Phi(i) \mu_{0}\left(\mathcal{R}_{0}-1\right) S(t)
\end{array}\right.
$$

For $\lambda \in \mathbb{C}$ such that $\operatorname{Re} \lambda>-\mu_{0}$ and looking for $S(t)=e^{\lambda t} s$ and $I(t, i)=z(i) e^{\lambda t}$ of the linearized problem (3.16), one gets

$$
\left\{\begin{array}{l}
\lambda s=-\mu_{0} \mathcal{R}_{0} s-\frac{S_{F}}{\mathcal{R}_{0}} \mathcal{T}(\beta z) \\
\lambda z(i)+(i \nu z(i))^{\prime}=-\mu(i) z(i)+\Phi(i)\left(\frac{\gamma}{\mu_{0}} \mathcal{T}(\beta z)+\mu_{0}\left(\mathcal{R}_{0}-1\right) s\right) .
\end{array}\right.
$$

The latter equation may be integrated and then applying the $\mathcal{T}(\beta \cdot)$ operator one deduces that $(s, \mathcal{T}(z))$ satisfies the linear system

$$
\left(\begin{array}{cc}
\left(\lambda+\mu_{0} \mathcal{R}_{0}\right) & \frac{\gamma}{\mu_{0} \mathcal{R}_{0}} \\
\mathcal{T}\left(\beta \pi_{\mu+\lambda}\right) \mu_{0}\left(\mathcal{R}_{0}-1\right) & \frac{\gamma \mathcal{T}\left(\beta \pi_{\mu+\lambda}\right)}{\mu_{0} \mathcal{R}_{0}}-1
\end{array}\right) \cdot\left(\begin{array}{c}
s \\
\mathcal{T}(\beta z)
\end{array}\right)=0 .
$$

Since $\mathcal{R}_{0}>1$, several computations lead to the characteristic equation for $\operatorname{Re} \lambda>-\mu_{0}$,

$$
\frac{\gamma \mathcal{T}\left(\beta \pi_{\mu+\lambda}\right)\left(\lambda+\mu_{0}\right)}{\mu_{0} \mathcal{R}_{0}\left(\lambda+\mu_{0} \mathcal{R}_{0}\right)}=1
$$

Let us denote by $\Gamma(\lambda)$ the left hand side of the previous equality. Considering the real and imaginary parts of $\lambda$ then identifying the real part of the equation $\Gamma(\lambda)=1$ gives

$$
\frac{\mu_{0} \mathcal{R}_{0}\left[\left(\operatorname{Re} \lambda+\mu_{0} \mathcal{R}_{0}\right)\left(\operatorname{Re} \lambda+\mu_{0}\right)+(\operatorname{Im} \lambda)^{2}\right]}{\left(\operatorname{Re} \lambda+\mu_{0}\right)^{2}+(\operatorname{Im} \lambda)^{2}}=\gamma \mathcal{T}\left(\frac{\beta(i)}{\nu i} \int_{i^{-}}^{i} \Phi(s) \cos (\operatorname{Im} \lambda \ln (i / s)) e^{-\int_{s}^{i} \frac{\operatorname{Re} \lambda+\mu(l)}{\nu l} d l} d s\right) .
$$


Using the equality $S_{*}=\frac{\gamma}{\mu_{0} \mathcal{R}_{0}}$, this latter equality can be also written as $\mu_{0}\left(\operatorname{Re} \lambda+\mu_{0}\right)\left(\mathcal{R}_{0}-1\right)=\left[\left(\operatorname{Re} \lambda+\mu_{0}\right)^{2}+(\operatorname{Im} \lambda)^{2}\right] \times$

$$
\left(S_{*} \mathcal{T}\left(\frac{\beta(i)}{\nu i} \int_{i^{-}}^{i} \Phi(s) \cos (\operatorname{Im} \lambda \ln (i / s)) e^{-\int_{s}^{i} \frac{\operatorname{Re} \lambda+\mu(l)}{\nu l} d l} d s\right)-1\right) .
$$

Suppose now that $\operatorname{Re} \lambda \geq 0$. Then one gets

$$
S_{*} \mathcal{T}\left(\frac{\beta(i)}{\nu i} \int_{i^{-}}^{i} \Phi(s) \cos (\operatorname{Im} \lambda \ln (i / s)) e^{-\int_{s}^{i} \frac{\operatorname{Re} \lambda+\mu(l)}{\nu l} d l} d s\right) \leq S_{*} \mathcal{T}\left(\beta \pi_{\mu}\right)=1 .
$$

Since $\mathcal{R}_{0}>1$, a contradiction yields from the last equation and (3.17). As a consequence, $A+D_{E_{*}} f$ always has a dominant eigenvalue with negative real part. Finally one deduces, as in the proof of Theorem 3.11 , that the endemic equilibrium $E_{*}$ is locally stable.

We now prove that the disease is uniformly strongly persistent under the assumption $\mathcal{R}_{-}>1$. We start by proving the uniform weak persistence of the disease. Suppose by contradiction that the disease is not uniformly-weakly persistent: then for a fixed $0<\varepsilon<\frac{\mu_{0}\left(\mathcal{R}_{-}-1\right)}{\|\beta\|_{\infty}}$, there exists initial conditions (that may depend on $\varepsilon$ ) such that $\sigma_{\rho}^{+}<\varepsilon$. The same arguments than ones developed to prove (3.9) then give $S^{-} \geq \frac{\gamma}{\mu_{0}+\|\beta\|_{\infty} \varepsilon}$, where $S^{-}$is defined in (3.8). Moreover, Applying the operator $\mathcal{T}$ to the transport equation in (1.2) one deduces, using the boundary condition, assumption (ii) on $\Phi$ and equation (3.5), that there exists $\tau>0$ such that for every $t>\tau, \sigma_{\rho}^{\prime}(t) \geq\left(-\|\mu\|_{\infty}+\beta_{0} S^{-}\right) \sigma_{\rho}(t)$. After an integration, one gets for every $t \geq \tau$,

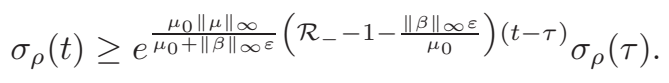

Since $\varepsilon<\frac{\mu_{0}\left(\mathcal{R}_{-}-1\right)}{\|\beta\|_{\infty}}$, one finally gets $\sigma_{\rho}^{+}=+\infty$, which contradicts $\sigma_{\rho}^{+}<\varepsilon$. Consequently, the disease is uniformly weakly persistent.

To prove that the disease persistence is uniformly strong, we now refer to a compactness argument that ensures that uniform weak persistence implies uniform strong persistence when there exists a compact attractor under the semiflow $\phi_{t}$ (see Thieme [43], Theorem A.32).

Using the equality (2.4) for the semiflow $\phi_{t}=\left(\phi_{t}^{S}, \phi_{t}^{I}\right)$ and the expression (3.14) of the semigroup, one deduces that the second component $\left.T_{A}(t-s)\left[f\left(\phi_{s}(x)\right)\right]\right|^{I}$ satisfies for every $x=\left(x^{S}, x^{I}\right) \in X_{+}$the equality

$$
\left.T_{A}(t-s)\left[f\left(\phi_{s}(x)\right)\right]\right|^{I}(i)=\phi_{s}^{S}(x) \mathcal{T}\left(\beta \phi_{s}^{I}(x)\right) \Phi\left(i e^{\nu(s-t)}\right) e^{-\int_{0}^{t-s}\left(\mu\left(i e^{-\nu \xi}\right)+\nu\right) d \xi} \chi_{\{\theta(i) \geq t-s\}} .
$$

Then an obvious change of variable implies that the component $\phi_{t}^{I}$ of the semiflow $\phi_{t}$ rewrites as $\phi_{t}^{I}(x)=$ $\phi_{1}(t, x, \cdot)+\phi_{2}(t, x, \cdot)$ with

$$
\begin{gathered}
\phi_{1}(t, x, i)=\left(x^{I}\left(i e^{-\nu t}\right) e^{-\int_{0}^{t}\left(\mu\left(i e^{-\nu s}\right)+\nu\right) d s}\right) \chi_{\{\theta(i) \geq t\}}, \\
\phi_{2}(t, x, i)=\int_{\mathbb{R}_{+}} \phi_{t-s}^{S}(x) \mathcal{T}\left(\beta \phi_{t-s}^{I}(x)\right) \Phi\left(i e^{-\nu s}\right) e^{-\int_{0}^{s}\left(\mu\left(i e^{-\nu \xi}\right)+\nu\right) d \xi} \chi_{\{0 \leq s \leq \min (\theta(i), t)\}} d s
\end{gathered}
$$

From here, consider the subset $B \subset X_{+}$defined by $B=\left\{\left(\phi_{t}^{S}(x), \phi_{2}(t, x, \cdot)\right), t \geq 0, x \in X_{0}\right\}$ where $X_{0}$ is defined in Lemma 3.2. We now prove that the adherence of $B$ in $\mathrm{X}$ is a compact attractor in the following sense:

1. $\bar{B}^{X}$ is a compact subset of $X$;

2. $\forall x \in X_{0}, \lim _{t \rightarrow+\infty} d\left(\phi_{t}(x), B\right)=0$ 
-1 . We start to prove the relative compactness of $B$. Since $\phi_{t}^{S}(x)$ is a real variable, we only need to consider the family $\left\{\phi_{2}(t, x, \cdot), t \geq 0, x \in X_{0}\right\}$ of $L^{1}(J)$. We identify $L^{1}(\mathbb{R})$ with the set of those functions that are zero on $\left(-\infty, i^{-}\right)$and we use the well-known Riesz-Frechet-Kolmogorov criterion (see for instance Yosida [49]). We leave to the reader easier conditions needed for the criterion, and concentrate the proof to check that the family $\left\{\phi_{2}(t, x, \cdot),(t, x) \in \mathbb{R}_{+} \times X_{0}\right\}$ satisfies

$$
\lim _{h \rightarrow 0} \sup _{(t, x) \in \mathbb{R}_{+} \times X_{0}}\left\|\tau_{h} \phi_{2}(t, x, \cdot)-\phi_{2}(t, x, \cdot)\right\|_{L^{1}}=0,
$$

where $\tau_{h}$ is the translation operator in $L^{1}$. Note that the limit above is then satisfied uniformly for $t \geq 0$ and for all solutions of the model. since $x \in X_{0}$ which is an invariant set for the semiflow as stated in Lemma 3.2 and $\theta$ is an increasing function, one gets by spliting the integral the following majoration,

$$
\left\|\tau_{h} \phi_{2}(t, x, \cdot)-\phi_{2}(t, x, \cdot)\right\|_{L^{1}} \leq\|\beta\|_{\infty}\left(\frac{\gamma}{\mu_{0}}\right)^{2}\left(\mathcal{I}_{1}(t, h)+\mathcal{I}_{2}(t, h)\right),
$$

with

$$
\mathcal{I}_{1}(t, h)=\iint_{J \times \mathbb{R}_{+}}\left|\Phi\left((i+h) e^{-\nu s}\right) e^{-\int_{0}^{s} \mu\left((i+h) e^{\nu \xi}\right) d \xi}-\Phi\left(i e^{-\nu s}\right) e^{-\int_{0}^{s} \mu\left(i e^{\nu \xi}\right) d \xi}\right| \chi_{\{s \leq \min (t, \theta(i)\}} d s d i
$$

and

$$
\mathcal{I}_{2}(t, h)=\iint_{J \times \mathbb{R}_{+}} \Phi\left((i+h) e^{-\nu s}\right) e^{-\int_{0}^{s} \mu\left((i+h) e^{\nu \xi}\right) d \xi} \chi_{\{\min (t, \theta(i)) \leq s \leq \min (t, \theta(i+h))\}} d s d i
$$

Since $\theta$ is an increasing function one gets $\{\min (t, \theta(i)) \leq s \leq \min (t, \theta(i+h))\} \subset\{\theta(i) \leq s \leq \theta(i+h)\}$, and the assumption (iii) on $\mu$ then implies that

$$
\mathcal{I}_{2}(t, h) \leq \iint_{J \times \mathbb{R}_{+}} \Phi\left((i+h) e^{-\nu s}\right) e^{-\mu_{0} s} \chi_{\{\theta(i) \leq s \leq \theta(i+h)\}} d s d i .
$$

Moreover, standard computations and majorations give

$$
\begin{aligned}
\mathcal{I}_{1}(t, h) \leq \iint_{J \times \mathbb{R}_{+}}\left|\Phi\left((i+h) e^{-\nu s}\right)-\Phi\left(i e^{-\nu s}\right)\right| e^{-\mu_{0} s} d s d i \\
\quad+\iint_{J \times \mathbb{R}_{+}} \Phi\left(i e^{-\nu s}\right)\left|e^{-\int_{0}^{s}\left(\mu\left((i+h) e^{\nu \xi}\right)+\nu\right) d \xi}-e^{-\int_{0}^{s}\left(\mu\left(i e^{\nu \xi}\right)+\nu\right) d \xi}\right| d s d i .
\end{aligned}
$$

From here, since assumptions (ii) and (iii) on $\Phi$ and $\mu$ imply that that the following functions are in $L^{1}\left(J \times \mathbb{R}_{+}\right)$,

$$
\begin{aligned}
& (i, s) \mapsto \Phi\left((i+h) e^{-\nu s}\right) e^{-\mu_{0} s} \chi_{\{\theta(i) \leq s \leq \theta(i+h)\}}, \\
& (i, s) \mapsto\left|\Phi\left((i+h) e^{-\nu s}\right)-\Phi\left(i e^{-\nu s}\right)\right| e^{-\mu_{0} s}, \\
& (i, s) \mapsto \Phi\left(i e^{-\nu s}\right)\left|e^{-\int_{0}^{s} \mu\left((i+h) e^{\nu \xi}\right) d \xi}-e^{-\int_{0}^{s} \mu\left(i e^{\nu \xi}\right) d \xi}\right|,
\end{aligned}
$$

one can apply the Lebesgue dominated convergence theorem in equations (3.20) and (3.21), proving that

$$
\lim _{h \rightarrow 0} \sup _{t \geq 0}\left(\mathcal{I}_{1}(t, h)+\mathcal{I}_{2}(t, h)\right)=0 .
$$

Equation (3.19) then proves that (3.18) is satisfied, and the relative compactness of $B$ yields. 
- 2. To prove this limit, we check that for $x \in X_{0}$,

$$
\begin{aligned}
\left\|\phi_{1}(t, x, \cdot)\right\|_{L^{1}} & =\int_{\theta(i) \geq t} x^{I}\left(i e^{-\nu t}\right) e^{-\int_{0}^{t}\left(\mu\left(i e^{-\nu s}\right)+\nu\right) d s} d i \\
& \leq \frac{\gamma}{\mu_{0}} e^{-\left(\mu_{0}+\nu\right) t}
\end{aligned}
$$

which ends the proof.

4. The numerical method. In this section we describe and study the discretization of (1.2). The scheme is then used in the next section to perform numerical simulations on the model applied to the transmission of prion pathologies.

4.1. The numerical scheme. We introduce an infection load-time grids where the infection load and the time steps are $\Delta i$ and $\Delta t$ respectively. We use the following discretizations $i_{j+1 / 2}=i^{-}+j \Delta i$, $t^{n}=n \Delta t$ where $j, n \in \mathbb{N}$. We define the cells $\left.K_{j}=\right] i_{j-1 / 2}, i_{j+1 / 2}\left[\right.$ centered at $i_{j}=\frac{1}{2}\left(i_{j-1 / 2}+i_{j+1 / 2}\right)$, for $j \geq 1$. We denote by $I_{j}^{n}$ the approximation of the average of $I\left(t^{n}, i\right)$ over the cell $K_{j}$, namely

$$
I_{j}^{n} \simeq \frac{1}{\Delta i} \int_{K_{j}} I\left(t^{n}, i\right) d i
$$

We use an implicit upwind finite volume scheme in order to compute $I_{j}^{n}$. The general scheme is as follows:

- We compute the initial states and the data:

$$
\begin{aligned}
& S^{0}=S(0), \quad I_{j}^{0}=\frac{1}{\Delta i} \int_{K_{j}} I_{0}(i) d i \\
& \beta_{j}=\frac{1}{\Delta i} \int_{K_{j}} \beta(i) d i, \mu_{j}=\frac{1}{\Delta i} \int_{K_{j}} \mu(i) d i \text { and } \Phi_{j}=\frac{1}{\Delta i} \int_{K_{j}} \Phi(i) d i .
\end{aligned}
$$

- Assume now $S^{n}$ and $I^{n}=\left(I_{j}^{n}\right)_{j \geq 1}$ are computed,

$\triangleright$ we define

$$
\mathcal{T}\left(\beta I^{n}\right)=\Delta i \sum_{j \geq 1} \beta_{j} I_{j}^{n}
$$

$\triangleright$ we compute

$$
S^{n+1}=\frac{\gamma \Delta t+S^{n}}{1+\Delta t\left(\mu_{0}+\alpha+\mathcal{T}\left(\beta I^{n}\right)\right)},
$$

$\triangleright$ we compute $I^{n+1}$ by solving the following linear system:

$$
-\nu \frac{\Delta t}{\Delta i} i_{j-1 / 2} I_{j-1}^{n+1}+\left(1+\nu \frac{\Delta t}{\Delta i} i_{j+1 / 2}+\Delta t \mu_{j}\right) I_{j}^{n+1}=I_{j}^{n}+\Delta t \Phi_{j} S^{n+1} \mathcal{T}\left(\beta I^{n}\right) .
$$

Let us now prove some basic properties of the scheme, namely the stability and that it preserves the positivity of the unknowns and it satisfies a balance equation.

Proposition 4.1. Assume that for any $n \geq 0, S^{n}$ and $I^{n}$ are computed by (4.2)-(4.3).

(i) For any $n \geq 0, j \geq 1$, we have $S^{n} \geq 0$ and $I_{j}^{n} \geq 0$.

(ii) Under the assumption $(H), S^{n}$ and $I^{n}$ satisfy

$$
\frac{S^{n+1}-S^{n}}{\Delta t}+\frac{\Delta i}{\Delta t} \sum_{j \geq 1}\left(I_{j}^{n+1}-I_{j}^{n}\right)=\gamma-\mu_{0} S^{n+1}-\mu_{1} \mathcal{T}\left(I^{n+1}\right),
$$

which is a discrete version of the balance equation (3.6). 
(iii) Let $T>0$. Then for any $t^{n} \leq T$, we have the following stability results:

$$
\begin{aligned}
& S^{n} \leq \gamma T+S^{0}, \\
& \mathcal{T}\left(I^{n}\right) \leq e^{T\|\beta\|_{\infty}\left(\gamma T+S^{0}\right)}\left(\mathcal{T}\left(I^{0}\right)+\alpha T\left(\gamma T+S^{0}\right)\right) .
\end{aligned}
$$

Proof. (i) Since $S_{0} \geq 0$ and $I_{0} \in L_{+}^{1}(J)$, then from (4.1), we have that $S^{0} \geq 0$ and for any $j \geq 1$, $I_{j}^{0} \geq 0$. Next an easy induction argument shows, from (4.2)-(4.3), that $S^{n} \geq 0$ and $I_{j}^{n} \geq 0$ for any $n \in \mathbb{N}$ and $j \geq 1$.

(ii) Observe that one can rewrite (4.2)-(4.3) into the form

$$
\begin{aligned}
& \frac{S^{n+1}-S^{n}}{\Delta t}=\gamma-\left(\mu_{0}+\alpha\right) S^{n+1}-S^{n+1} \mathcal{T}\left(\beta I^{n}\right), \\
& \frac{I_{j}^{n+1}-I_{j}^{n}}{\Delta t}+\frac{1}{\Delta i}\left(\nu i_{j+1 / 2} I_{j}^{n+1}-\nu i_{j-1 / 2} I_{j-1}^{n+1}\right)=-\mu_{j} I_{j}^{n+1}+\Phi_{j} S^{n+1} \mathcal{T}\left(\beta I^{n}\right) .
\end{aligned}
$$

This leads to

$$
\begin{aligned}
& \frac{S^{n+1}-S^{n}}{\Delta t}+\frac{\Delta i}{\Delta t} \sum_{j \geq 1}\left(I_{j}^{n+1}-I_{j}^{n}\right) \\
& =\gamma-\left(\mu_{0}+\alpha\right) S^{n+1}-S^{n+1} \mathcal{T}\left(\beta I^{n}\right)+\nu i_{1 / 2} I_{0}^{n+1}-\Delta i \sum_{j \geq 1} \mu_{j} I_{j}^{n+1}+\mathcal{T}\left(\beta I^{n}\right) S^{n+1} \Delta i \sum_{j \geq 1} \Phi_{j} .
\end{aligned}
$$

Then the discrete balance equation (4.4) follows from the boundary condition of (1.2) which implies that $\nu i_{1 / 2} I_{0}^{n+1}=\alpha S^{n+1}$, from the fact that $\Delta i \sum_{j \geq 1} \Phi_{j}=\int_{J} \Phi(i) d i=1$ and from assumption $(H)$ that implies $\mu_{j}=\mu_{1}$, for any $j \geq 1$.

(iii) From (4.2), we clearly have $S^{n} \leq\left(\gamma \Delta t+S^{n-1}\right)$, for any $n \geq 1$. Then we can write $S^{n} \leq 2 \gamma \Delta t+S^{n-2}$ and by induction, for any $n \geq 1$, such that $n \Delta t \leq T$ we obtain (4.5).

Now, summing the second equation of (4.7) over $j \geq 1$, we get

$$
\sum_{j \geq 1} \frac{I_{j}^{n+1}-I_{j}^{n}}{\Delta t}-\frac{\nu}{\Delta i} i_{1 / 2} I_{0}^{n+1}=-\sum_{j \geq 1} \mu_{j} I_{j}^{n+1}+S^{n+1} \mathcal{T}\left(\beta I^{n}\right) \sum_{j \geq 1} \Phi_{j} .
$$

Using the same arguments as in (ii), we arrive at

$$
\sum_{j \geq 1}\left(1+\Delta t \mu_{j}\right) I_{j}^{n+1}=\frac{1}{\Delta i} \mathcal{T}\left(I^{n}\right)+\frac{\Delta t}{\Delta i} S^{n+1} \mathcal{T}\left(\beta I^{n}\right)+\frac{\Delta t}{\Delta i} \alpha S^{n+1} .
$$

Therefore, we have

$$
\mathcal{T}\left(I^{n+1}\right) \leq\left(1+\Delta t\|\beta\|_{\infty} S^{n+1}\right) \mathcal{T}\left(I^{n}\right)+\Delta t \alpha S^{n+1} .
$$

It follows from (4.5) that

$$
\mathcal{T}\left(I^{n+1}\right) \leq\left(1+\Delta t\|\beta\|_{\infty}\left(\gamma T+S^{0}\right)\right) \mathcal{T}\left(I^{n}\right)+\Delta t \alpha\left(\gamma T+S^{0}\right) .
$$

Using discrete version of Gronwall's lemma we obtain (4.6).

\section{口}


4.2. Comparison with explicit solutions. A possibility to validate the scheme is to compare the numerical solutions with explicit available solutions. Since no explicit solutions are available for the complete model (1.2), we consider the particular case of system (3.4) where we assume $\beta(i)=0$ f.a.e. $i$ in $J$. Hence (3.4) is reduced to the following system of ODE

$$
\left\{\begin{array}{l}
\frac{d S(t)}{d t}=\gamma-\left(\mu_{0}+\alpha\right) S(t) \\
\frac{d \mathcal{T}(I)(t)}{d t}=\alpha S(t)-\mu_{1} \mathcal{T}(I)(t) \\
S(0)=S_{0}, \quad \mathcal{T}(I)(0)=\mathcal{T}\left(I_{0}\right)
\end{array}\right.
$$

For convenience, we assume $\mu_{0}+\alpha-\mu_{1} \neq 0$. Then system (4.8) has a unique pair of solution $(S(t), \mathcal{T}(I)(t))$ defined by

$$
S(t)=\left(S_{0}-\frac{\gamma}{\mu_{0}+\alpha}\right) e^{-\left(\mu_{0}+\alpha\right) t}+\frac{\gamma}{\mu_{0}+\alpha}
$$

and

$$
\mathcal{T}(I)(t)=\mathcal{T}\left(I_{0}\right) e^{-\mu_{1} t}+\frac{\alpha}{\mu_{0}+\alpha-\mu_{1}}\left(S_{0}-\frac{\gamma}{\mu_{0}+\alpha}\right)\left(e^{-\mu_{1} t}-e^{-\left(\mu_{0}+\alpha\right) t}\right)+\frac{\alpha \gamma}{\mu_{1}\left(\mu_{0}+\alpha\right)}\left(1-e^{-\mu_{1} t}\right) .
$$

We now consider the following arbitrary parameters: $\mu_{0}=0.1, \mu_{1}=0.15, \nu=0.1, \alpha=0.02, \gamma=1$. The initial conditions are assumed to be $S_{0}=100$ and $\mathcal{T}(I)(0)=0.1$. In order to reduce the computation, we truncate the domain by assuming that $i^{-} \leq i \leq i^{+}$and $0 \leq t \leq T$, where we set $i^{-}=0.1, i^{+}=100$ and $T=50$. The infected load grid $\Delta i$ is fixed to $\Delta i=0.05$. We define the following relative $L^{1}$-discrete error norms

$$
e^{S}=\frac{\sum_{0 \leq n \leq N}\left|S\left(t^{n}\right)-S^{n}\right|}{\sum_{0 \leq n \leq N}\left|S\left(t^{n}\right)\right|}, e^{\mathcal{T}}=\frac{\sum_{0 \leq n \leq N}\left|\mathcal{T}(I)\left(t^{n}\right)-\mathcal{T}\left(I^{n}\right)\right|}{\sum_{0 \leq n \leq N}\left|\mathcal{T}(I)\left(t^{n}\right)\right|},
$$

where $N$ is an integer such that $T=N \Delta t$.

In Figure 4.1, we report the $L^{1}$-discrete error norms $e^{S}$ and $e^{\mathcal{T}}$ using logarithmic scale. We deduce the orders of convergence for each variables, which are respectively 1 for $S$ and 0.68 for $\mathcal{T}(I)$. Note that these orders can be improved using more accurate schemes (see e.g. [25, 38]). On Figure 4.2, both explicit and numerical solutions are plotted. For the numerical solutions we use the time step $\Delta t=0.025$. We observe good agreements.

5. Application: transmission of prion pathologies. Prion pathologies, such as Scrapie or BSE, are fatal-issued diseases characterized by variable incubation periods during which the disease cannot be detected. At the end of the incubation period, infected individuals usually develop distinctive clinical signs which are rapidly followed by death. As a consequence, no recovery is possible.

Modeling the same way as in $[18,41]$, incubation time heterogeneity may be incorporated in the model through the infection load variable $i$. Indeed, Equation (1.3) on $\mu$ implies that the quantity $\Pi: J \rightarrow(0,1]$ given by

$$
\Pi(i)=e^{-\int_{i-}^{i} \mu(l) d l}
$$



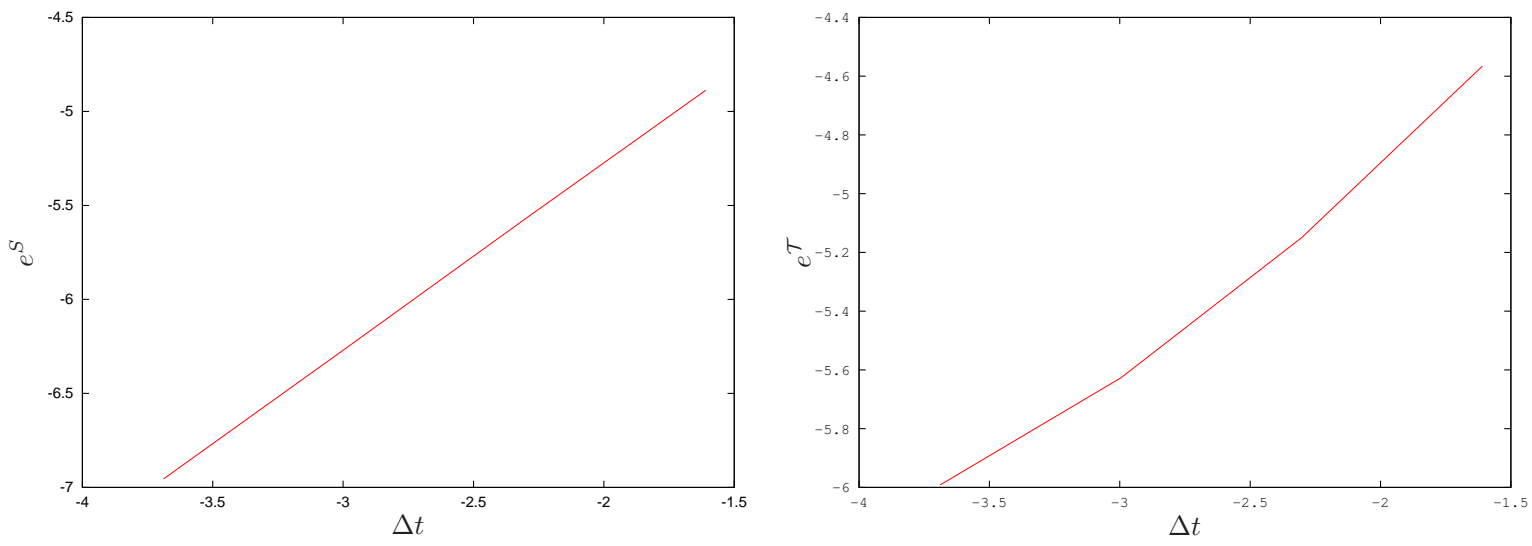

FIG. 4.1. $L^{1}$ relative errors norms $e^{S}$ and $e^{\mathcal{T}}$ in $\log / \log$ scale

denotes the probability to survive for an infected individual with infection load $i \in J$. Consider now a given threshold $\Pi_{d} \in(0,1)$ such that an individual with an infection load that satisfies $\Pi(i) \leq \Pi_{d}$ is considered to be dead or about to die, meaning that the incubation time of the disease is gone. Since $\Pi$ is a strictly decreasing function, there is a unique $i_{d}>i^{-}$such that $\Pi\left(i_{d}\right)=\Pi_{d}$. Then for an initial infection load at contamination $i_{0} \in\left(i^{-}, i_{d}\right)$ the incubation time $\tau$ is given by

$$
\tau=\frac{1}{\nu} \ln \left(\frac{i_{d}}{i_{0}}\right) .
$$

In other words, the incubation time $\tau$ represents the time for the infectious process to reach the value $i_{d}$ from the initial value $i_{0}$. Then the map $i_{0} \mapsto \tau\left(i_{0}\right)$ defines a natural change of variable between incubation time of the disease and initial infection loads at contamination and enables us to incorporate in the model the variable incubation times using the distribution $\Phi$.

The goal of this section is to perform simulations that either allow to strengthen our theoretical results or to show that these latter could be improved. To achieve that goal, we focus on a specific prion pathology that is scrapie: indeed, Problem (1.2) is a variation of scrapie models that describe the propagation of the disease in flocks $[18,31,32,41,45]$, where the age-structure and the genotype specificity are avoided. More precisely, the following table sums up the specific characteristics of scrapie and why they are well-translated by the model:

\section{Characteristics}

No recovery

Disease with fatal issue

Exponential growth of the infection

Variable incubation time at contagion

External source of contamination

$I$ infectious

\section{Model}

states $S$ (susceptible) and $I$ (infected)

infection-load dependent mortality rate $\mu(i)$ for the class $I$ with assumption (iii)

infection load $i \in J$ that satisfies $d i / d t=\nu i$ distributed initial infection load with p.d.f. $\Phi$ System input with contamination rate $\alpha$ force of infection $\propto \mathcal{T}(I)=\int_{J} I(i) d i$ 

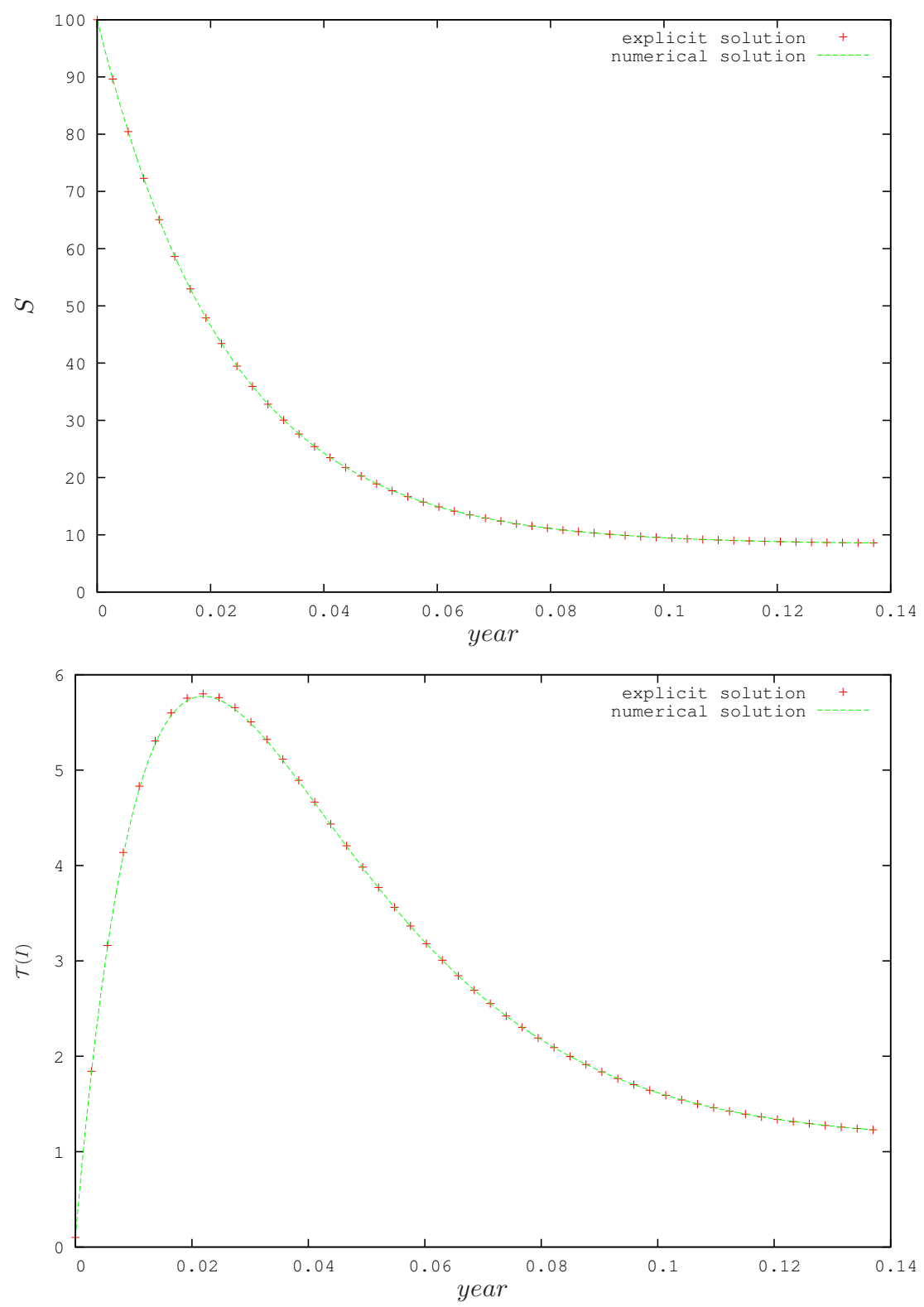

FIG. 4.2. Comparison between explicit solutions and computed solutions

The distribution of initial infection load $\Phi$ is choosed, according to [41], as a $\Gamma$ distribution of parameters $(g, c)$, that is

$$
\Phi(i)=\frac{i^{c-1} e^{-\frac{i}{g}}}{\Gamma(c) g^{c}} .
$$

The mean and standard deviation, that caracterize the distribution $\Phi$, are given by $m_{\Phi}=g c$ and $\sigma_{\Phi}=g^{2} c$. Parameters $g$ and $c$ in Table 5.1 are computed to ensure that $m_{\Phi}=0.35$ and $\sigma_{\Phi}=0.05$, as in [32].

In [41], the natural mortality rate is an age-dependent function $\mu(a)$ given by a Weibull distribution: 
TABLE 5.1

Parameter values used for the simulations.

\begin{tabular}{lcc}
\hline \hline Parameter definition & symbol & value \\
\hline \hline initial susceptible population size & $S_{0}$ & 10 indiv. \\
initial infected load range & {$\left[i^{-}, i^{+}\right]$} & {$\left[10^{-2}, 10^{2}\right]$ years } \\
basic mortality rate & $\mu_{0}$ & 0.39 year $^{-1}$ \\
horizontal transmission rate & $\beta$ & $4.10^{-2}{\text { (indiv. } \cdot \text { year })^{-1}}^{-1}$ \\
infection load velocity & $\nu$ & 1.15 year $^{-1}$ \\
contamination rate & $\alpha$ & 0 year $^{-1}$ \\
first infection load distribution $\Phi$ & $(g ; c)$ & $\left(7.10^{-3} ; 49\right)$ \\
\hline \hline & Scenario 1 \\
\hline initial infected population size & $\mathcal{T}\left(I_{0}\right)_{1}$ & 1 indiv. $^{-1}$ \\
entering flux & $\left(\gamma_{1} ; \tilde{\gamma}_{1}\right)$ & $(10 ; 7)$ indiv.year $^{-1}$ \\
\hline \hline
\end{tabular}

$\mu(a)=0.22^{2} a$, with the average natural lifespan that is $\bar{a}=4$ years. The value of $\mu_{0}$ in Table 5.1 is then computed from the weibull distribution and the value $\bar{a}$. From here, the infection mortality rate is taken as $\mu(i)=\mu_{0}\left(2-\frac{1+i^{-2}}{1+i^{2}}\right)$, so that assumption (iii) is satisfied.

The entering flux $\gamma$, choosed in order to simulate different scenarios, allows to compute some values of $\mathcal{R}_{0}, \mathcal{R}_{+}$or $\mathcal{R}_{-}$that are greater or smaller that 1 . In both following scenarios the model is considered without external source of contamination so that $\alpha=0$.

Scenario 1. This scenario illustrates the case of disease persistence. Corresponding to the value $\gamma_{1}=10$ we have $\mathcal{R}_{-} \approx 1.31$. According to Theorem 3.12, one can check on Figures 5.1 that, even if the initial data of infected is small $\left(\mathcal{T}\left(I_{0}\right)_{1}=1\right)$, the disease is persistent. Moreover, another value of the entering flux is choosed in this scenario to test if the disease may persist when $\mathcal{R}_{-}<1<\mathcal{R}_{0}$. Taking the value $\tilde{\gamma}_{1}=7$ for the enterring flux, one gets $\mathcal{R}_{-} \approx 0.92$ whereas $\mathcal{R}_{0} \approx 1.17$. In that case, one can see that, although keeping a small intial data for the infected population, the persistence of the disease remains.
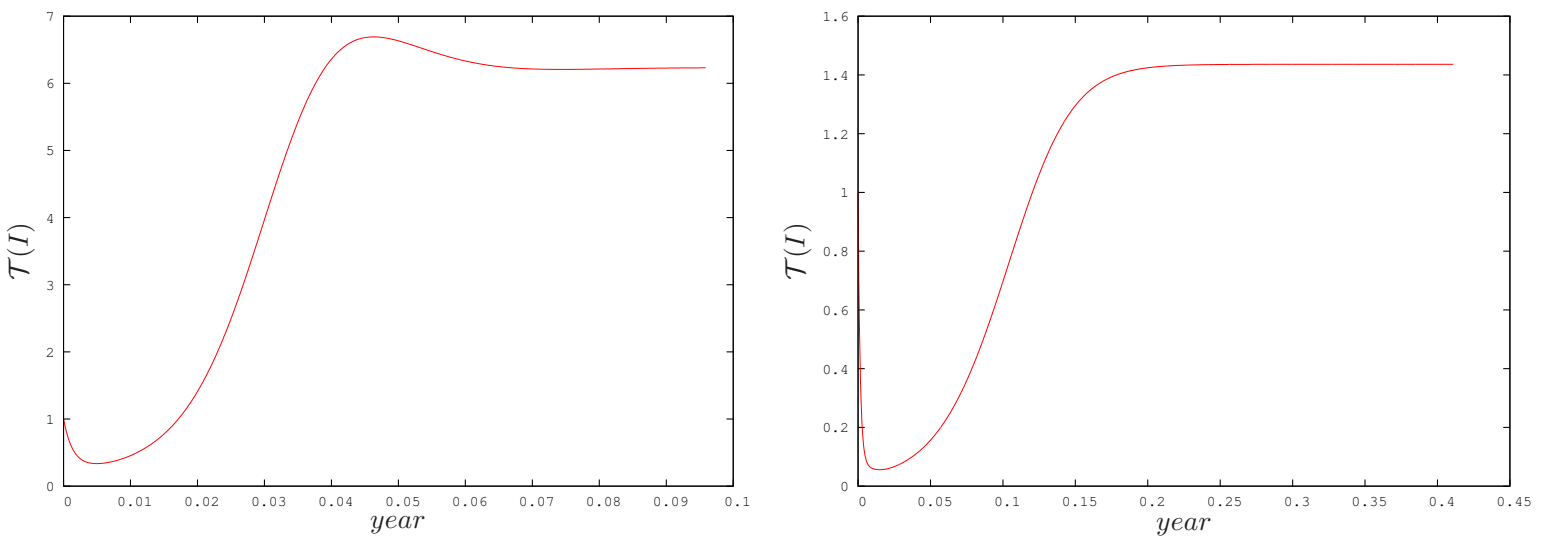

FIG. 5.1. Scenario 1- Total infected curve for $\mathcal{R}_{-}>1$ (left) and $\mathcal{R}_{-}<1<\mathcal{R}_{0}$ (right)

Scenario 2. This scenario illustrates the case of disease extinction. The parameter value $\gamma_{2}$, resp. $\tilde{\gamma}_{2}$, of the entering flux corresponds to the case where $\mathcal{R}_{+}<1$, resp. $\mathcal{R}_{0}<1<\mathcal{R}_{+}$(see Figures 5.2). For these simulations, a big initial data for the infected population is choosed in proportion of the overall population $(\approx 60 \%)$, confirming the global stability of the disease free equilibrium. Indeed, the infected 
curve (red color) converges to 0 , while the susceptible curve (green color) tends to stabilize around the equilibrium point. Moreover, the value $\tilde{\gamma}_{2}$ yields $\mathcal{R}_{+} \approx 1.31$ and $\mathcal{R}_{0} \approx 0.84$. In that particular case, Figure 5.2 (right) also suggests that the global stability of the disease free equilibrium is satisfied when $\mathcal{R}_{0}<1$.

$(a)$

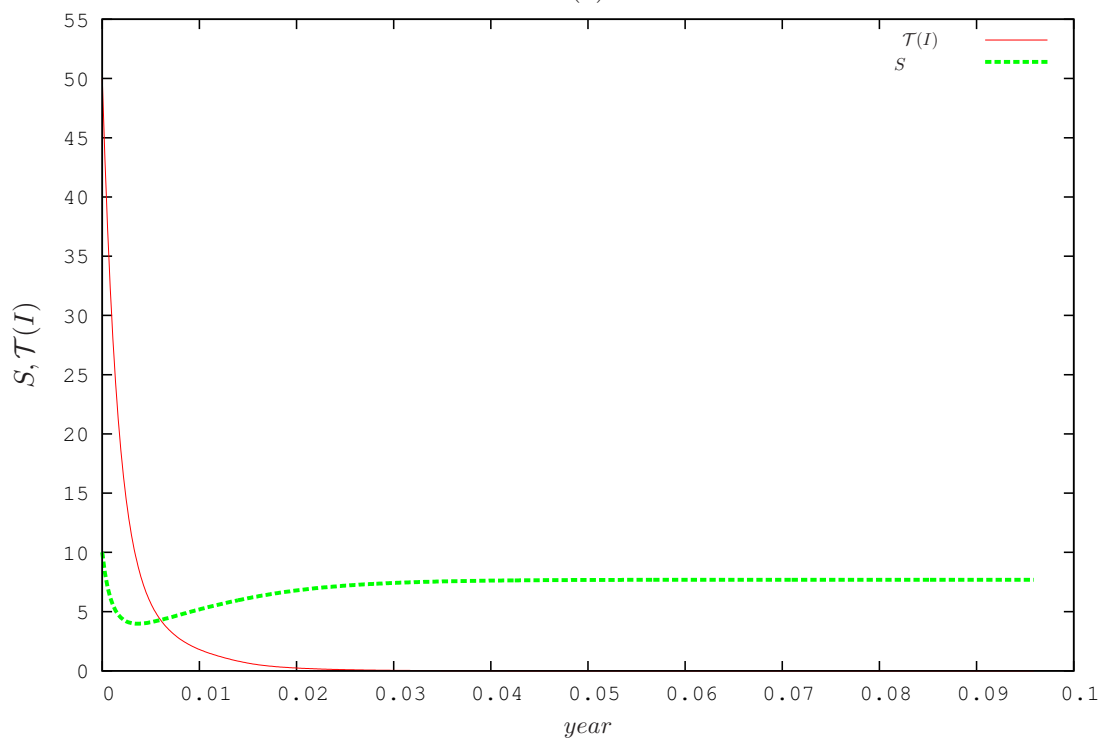

(b)

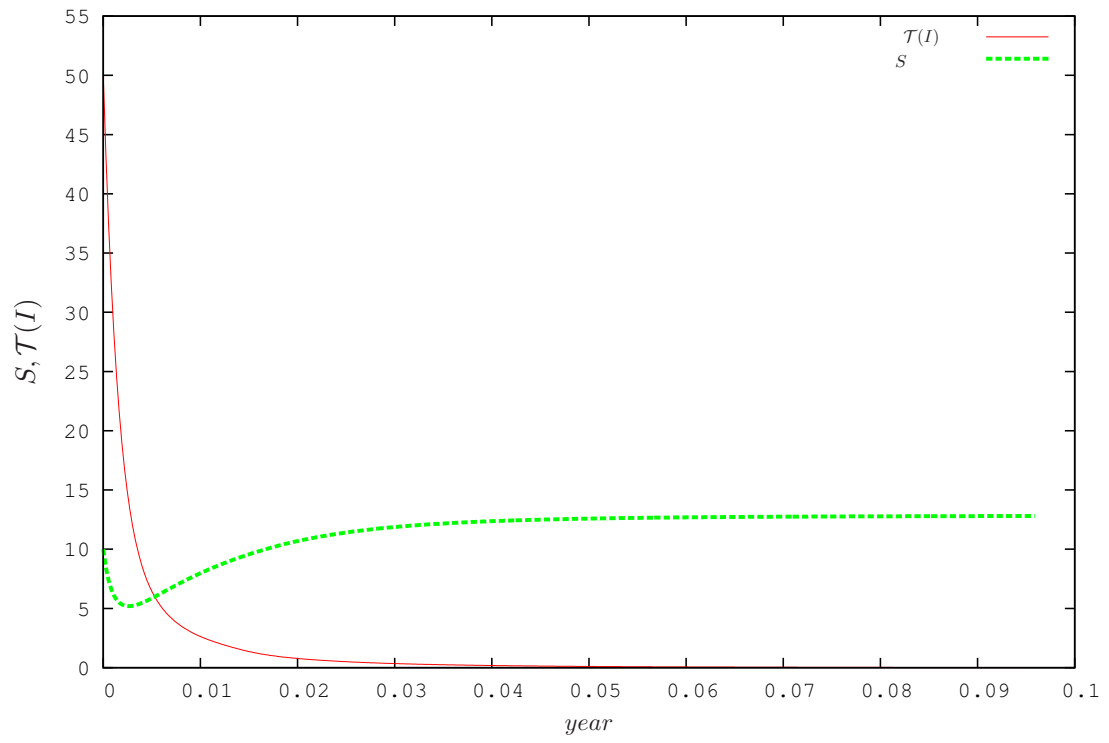

FIG. 5.2. Scenario 2- Total infected curve for $\mathcal{R}_{+}<1$ (a) and $\mathcal{R}_{0}<1<\mathcal{R}_{+}$(b)

6. Final remarks. In this paper, we have studied the asymptotic behavior of the solution of an infection load-structured SI model. When the model incorporates a constant external contamination, we proved by the use of fluctuation methods that the disease is uniformly strongly persistent, and exhibit a lower uniform bound for the infected population. In the case whithout external contamination, we proved 
that the model exhibits the traditional threshold behavior where the disease-free equilibrium is locally asymptotically stable if the basic reproduction number is less than one, and the endemic equilibrium is locally asymptotically stable if the basic reproduction number is greater than one. These stability results are proved using semigroup theory. Moreover, linked to the basic reproduction number $\mathcal{R}_{0}$, the value $\mathcal{R}_{-}$, resp. $\mathcal{R}_{+}$, ensure that the disease is uniformly strongly persistent when $\mathcal{R}_{-}$is greater than one, resp. the disease-free equilibrium is globally stable if $\mathcal{R}_{+}$is less than one. These consistency results are proved using fluctuation methods coupled with compactness arguments.

A numerical scheme adapted to the model is built and analyzed. We then show that such an infection loadstructured model can be applied to the transmition of prion pathologies and use the numerical scheme to perform simulations. The different scenario we proposed illustrate well the theoretical results that are proved in the paper. Furthermore, some simulations make us think that the consistency theoretical results that are proved with values $\mathcal{R}_{-}>1$ and $\mathcal{R}_{+}<1$ should remain by substituting the thresholds $\mathcal{R}_{-}$and $\mathcal{R}_{+}$by $\mathcal{R}_{0}$.

Future works will be dedicated to the analyzis of the global stability of the disease-free equilibrium when $\mathcal{R}_{0}<1$ and of the endemic equilibrium when $\mathcal{R}_{0}>1$, on the basis of similar results proved in the case of SI models structured according to age of infectivity [27, 28]. Another future work will be the study of a model incorporating a more general external source of contamination. For instance, in the particular case of prion pathologies, the food intake would lead to a linear but non-autonomous and periodic boundary condition. Some other cases, such as an environmental spatially localized source of contamination, would be modeled by a non-linear function of the density $S(t)$ with a finite limit translating a saturation process of the number of susceptibles in contact with the source. This would lead to formulate a PDE problem with a non-linear boundary condition that may take into consideration more realistic cases than with a linear external source of contamination.

\section{REFERENCES}

[1] R.M. Anderson and R.M. May, Infectious Diseases of Humans: Dynamics and control, Oxford University Press, Oxford, 1991.

[2] W. Arendt, Resolvent positive operators, Proc. London Math. Soc. 54 (1987), pp. 321-349.

[3] O. ARINo, Some spectral properties for the asymptotic behavior of semigroups connected to population dynamics, SIAM Rev., 34(4) (1992), pp. 445-476.

[4] O. Arino, A survey of structured cell population dynamics, Acta Biotheoretica, 43 (1995), pp. 3-25.

[5] N. T. J. BAiley, The mathematical Theory of Epidemics, Charles Griffin \& Co, 1957.

[6] F. Brauer and C. Castillo-Chavez, Mathematical Models in Population Biology and Epidemiology, Springer, New York, 2000.

[7] G. Butler and P. Waltman, Persistence in dynamical systems, J. Differ. Equations, 63 (1986), pp. $255-263$.

[8] J. Burton, L. Billings, A.T. Derek and I.B. Schwartz, Disease persistence in epidemiological models: The interplay between vaccination and migration, Math. Biosci., 239(1) (2012), pp. 91-96.

[9] V. Capasso, Mathematical Structures of Epidemic Systems, Lecture Notes in Biomathematics, Vol. 97, Springer-Verlag, Heidelberg, 1993.

[10] T. Cazenave and A. Haraux, An Introduction to Semilinear Evolution Equations, Oxford Science Publication, Oxford, 2006.

[11] J.M. Cushing, An Introduction to Structured Population Dynamics, SIAM, Philadelphia, 1998.

[12] T. Dhirasakdanon, H.R. Thieme and P. Van Den Driessche, A sharp threshold for disease persistence in host metapopulations, Journal of Biological Dynamics 1(4) (2007), pp. 363-378.

[13] O. Diekmann and J.A.P. Heersterbeek, Mathematical Epidemiology of Infectious Diseases, Wiley Series in Mathematical and computational biology, Wiley, Chichester, 2000.

[14] L. Edelstein-Keshet, Mathematical Models in Biology, Classics in Applied Mathematics 46, SIAM, 2005.

[15] K.J. Engel and R. Nagel, One-Parameter Semigroups for Linear Evolution Equations, Graduate Texts in Mathematics, Springer, 2000.

[16] K.J. Engel And R. NAGel, A short course on operator semigroups, Universitext, Springer, 2006.

[17] H.I. Freedman and P. Moson, Persistence definitions and their connections, Proc. Amer. Soc., 109 (1990), pp. 10251033.

[18] T.J. Hagenaars, C.A. Donnelly, N.M. Ferguson, R.M. Anderson, The transmission dynamics of the aetiological agent of scrapie in a sheep flock, Math. Biosci., 168(2) (2000), pp. 117-135. 
[19] T.J. Hagenaars, C.A. Donnelly and N.M. Ferguson, Spatial heterogeneity and the persistence of infectious diseases, J. Theor. Biol., 229 (2004), pp. 349-359.

[20] W. Hirsch, H. Hanisch and J.P. Gabriel, Differential equation models for some parasitic infections: Methods for the study of asymptotic behavior, Comm. Pure Appl. Math., 38 (1985), pp. 733-753.

[21] J.M. Ireland, B.D. Mestel and R.A. Norman, The effect of seasonal host birth rates on disease persistence, Math. Biosci., 206(1) (2007), pp. 31-45.

[22] W. O. Kermack and A. G. McKendrick, A Contribution to the Mathematical Theory of Epidemics, Proc. R. Soc. Lond. Ser. A, 115 (1927), pp. 700-721.

[23] W. O. Kermack and A. G. McKendrick, Contributions to the Mathematical Theory of Epidemics: II, Proc. R. Soc. Lond. Ser. B, 138 (1932), pp. 55-83.

[24] W. O. Kermack and A. G. McKendrick, Contributions to the Mathematical Theory of Epidemics: III, Proc. R. Soc. Lond. Ser. B, 141 (1933), pp. 94-112.

[25] R. J. Leveque, Finite volume methods for hyperbolic problems, Cambridge Texts in Applied Mathematics. Cambridge University Press, Cambridge, 2002.

[26] X. LiU And P. Stechlinski, Infectious disease models with time-varying parameters and general nonlinear incidence rate, Appl. Math. Model., 36 (2012), pp. 1974-1994.

[27] P. Magal, C.C. McCluskey and G.F. WebB, Lyapunov functional and global asymptotic stability for an infection-age model, Appl. Anal. 89(7) (2010), pp. 1109-1140.

[28] P. Magal and C.C. MCCluskey, Two group infection age model: an application to nosocomial infection, SIAM J. Appl. Math., 73(2) (2013), pp. 1058-1095.

[29] J.D. Murray, Mathematical Biology I : An Introduction, Interdisciplinary Applied Mathematics 17, Springer, 2004.

[30] A. PAZY, Semigroups of linear operators and applications to partial differential equations, Applied Mathematical Sciences 44, Springer, 1983.

[31] A. Perasso And B. Laroche, Well-posedness of an epidemiological model described by an evolution PDE, ESAIM:PROC. 25 (2008), pp. 29-43.

[32] A. Perasso, B. Laroche, Y. Chitour and S. Touzeau, Identifiability analysis of an epidemiological model in a structured population, J. Math. Anal. Appl., 374 (2011), pp. 154-165.

[33] A. Perasso and U. Razafison, Infection Load Structured SI Model With Exponential Velocity And External Source of Contamination, Proceedings of the International Conference of Applied and Engineering Mathematics, WCE London, 2013, available online at http://www.iaeng.org/publication/WCE2013/WCE2013_pp263-267.pdf.

[34] B. Perthame, Transport Equations in Biology, Birkhäuser, 2010.

[35] J. Prüss, Equilibrium solutions of Age-Specific Populations Dynaics of several Species, J. Math. Biol., 84 (1981), pp. 65-84.

[36] R. Rosà, A. Pugliese, R. Norman and P.J. Hudson, Thresholds for disease persistence in models for tick-borne infections including non-viraemic transmission, extended feeding and tick aggregation, J. Theor. Biol., 224 (2003), pp. 359-376.

[37] I. SEgAL, Non-linear semi-groups, Ann. Math., 78 (1963), pp. 339-364.

[38] C.-W. Shu, Essentially Non-Oscillatory and Weighted Essentially Non-Oscillatory Schemes for Hyperbolic Conservation Laws, In Advance Numerical Approximation of Nonlinear Hyperbolic Equations, Lecture Notes in Mathematics, Vol. 1697, Springer, Berlin,1998, pp. 325-432.

[39] H.L. Smith And H.R. Thieme, Dynamical systems and population persistence, American Mathematical Society, Graduate Studies in Mathematics 118, 2011.

[40] H.L. Smith And H.R. Thieme, Persistence of bacteria and phages in a chemostat, J. Math. Biol., 64(6) (2012), pp. 951-979

[41] S.M. Stringer, N. Hunter And M.E.J. Woolhouse, A mathematical model of the dynamics of scrapie in a sheep flock, Math. Biosci., 153(2) (1998), pp. 79-98.

[42] H.R. Thieme And C. Castillo-Chavez, How may infection-age-dependent infectivity affect the dynamics of HIV/AIDS ?, SIAM J. Appl. Math., 53(5) (1993), pp. 1447-1479.

[43] H.R. Thieme, Mathematics in Population Biology, Princeton Series in Theoretical and Computational Biology, Princeton, 2003.

[44] H.R. Thieme, A. Tridane And Y. Kuang, An epidemic model with post-contact prophylaxis of distributed length I. Threshold for disease persistence and extinction, J. Biol. Dyn., 2 (2008), pp. 221-239.

[45] S. Touzeau, M.E. Chase-Topping, L. Matthews, D. Lajous, F. Eychenne, N. Hunter, J.D. Foster, G. Simm, J.-M. Elsen And M.E.J. Woolhouse, Modelling the spread of scrapie in a sheep flock: evidence for increased transmission during lambing seasons, Arch. Virol., 151 (2006), pp. 735-751.

[46] G.F. WebB, Theory of Nonlinear Age-Dependent Population Dynamics, Marcel Dekker, New York, 1985.

[47] G.F. WebB, Dynamics of populations structured by internal variables, Math. Z., 189 (1985), pp. 319-335.

[48] G.F. WebB, Population models structured by age, size, and spatial position, Structured Population Models in Biology and Epidemiology, Lecture Notes in Mathematics, Vol. 1936, Springer, Berlin, 2008, pp. 1-49.

[49] K. Yosida, Functional Analysis, Classics in Mathematics, Springer-Verlag, 1995. 